\title{
A NEW APPROACH FOR MODELING AND CONTROL OF NONLINEAR SYSTEMS VIA NORM-BOUNDED LINEAR DIFFERENTIAL INCLUSIONS
}

\author{
Roman Kuiava* \\ kuiava@eletrica.ufpr.br
}

\author{
Rodrigo A. Ramos ${ }^{\dagger}$ \\ ramosesc.usp.br
}

\author{
Hemanshu R. Pota \\ h.pota@adfa.edu. au
}

${ }^{*}$ Federal University of Parana (UFPR), Polytechnic Center, Department of Electrical Engineering, Rua Cel. Francisco Heraclito dos Santos, 100, Jardim das Americas, 81531-980, Curitiba, Brazil

${ }^{\dagger}$ University of Sao Paulo (USP), Engineering School of Sao Carlos (EESC), Department of Electrical Engineering, Av. Trabalhador Saocarlense, 400, Centro, 13566-590, Sao Carlos, Brazil

$\ddagger$ University of New South Wales at Australian Defence Force Academy (UNSW@ADFA), School of Information Technology and Electrical Engineering, Northcott Drive, Canberra, Australia

\section{RESUMO}

Uma nova abordagem para modelagem e controle de sistemas não-lineares via inclusões diferenciais lineares limitadas por norma

Este artigo propõe um método sistematico para modelagem de sistemas não-lineares na forma de inclusões diferenciais limitadas por norma (IDLNs). O modelo de IDLN resultante é adequado para aplicação de técnicas de projeto de controle linear, o que possibilita atender critérios específicos de desempenho dinâmico para o sistema não linear original em uma região de operação de interesse no espaço de estados, a partir de um controlador linear projetado para a sua representação na forma de IDLN. Então, um procedimento para projeto de um controlador por realimentação dinâmica de saída para um sistema descrito na forma de IDLN é também proposto neste artigo. Uma das principais contribuições da abordagem proposta de modelagem e controle é a aplicação do teorema do valor intermediário para representar sistemas não-lineares na forma de um modelo linear com parâmetros variantes no tempo, o qual é então mapeado em uma inclusão diferencial linear politópica (IDLP).

Artigo submetido em 24/03/2011 (Id.: 1308)

Revisado em 05/05/2011, 24/08/2011, 06/09/2011

Aceito sob recomendação do Editor Associado Prof. Daniel Coutinho
Para evitar o problema combinatório inerente aos modelos politópicos para sistemas de médio e grande porte, a IDLP é transformada em uma IDLN, e este processo é feito de tal forma que todas as trajetórias do sistema não-linear original sejam também trajetórias do modelo resultante de IDLN. Além do mais, é também possível escolher uma estrutura particular para os parâmetros da IDLN de forma à reduzir o conservadorismo na representação do sistema não-linear pelo modelo de IDLN, e esta característica é também uma importante contribuição deste artigo. Quanto ao projeto do controlador, ele é formulado como um problema de busca por uma solução que satisfaça um conjunto de restrições escritas na forma de desigualdades matriciais bilineares (ou BMIs, do inglês bilinear matrix inequalities). Tal solução é então encontrada usando-se um procedimento de separação em duas etapas que transforma o conjunto original de BMIs em um conjunto correspondente de desigualdades matriciais lineares (ou LMIs, do inglês linear matrix inequalities). Dois exemplos numéricos são apresentados para demonstrar a eficiência da abordagem proposta.

PALAVRAS-CHAVE: sistemas não-lineares; controle por realimentação dinâmica de saída; inclusões diferenciais lineares; controle robusto; desigualdades matriciais lineares. 


\section{ABSTRACT}

A systematic approach to model nonlinear systems using norm-bounded linear differential inclusions (NLDIs) is proposed in this paper. The resulting NLDI model is suitable for the application of linear control design techniques and, therefore, it is possible to fulfill certain specifications for the underlying nonlinear system, within an operating region of interest in the state-space, using a linear controller designed for this NLDI model. Hence, a procedure to design a dynamic output feedback controller for the NLDI model is also proposed in this paper. One of the main contributions of the proposed modeling and control approach is the use of the meanvalue theorem to represent the nonlinear system by a linear parameter-varying model, which is then mapped into a polytopic linear differential inclusion (PLDI) within the region of interest. To avoid the combinatorial problem that is inherent of polytopic models for medium- and large-sized systems, the PLDI is transformed into an NLDI, and the whole process is carried out ensuring that all trajectories of the underlying nonlinear system are also trajectories of the resulting NLDI within the operating region of interest. Furthermore, it is also possible to choose a particular structure for the NLDI parameters to reduce the conservatism in the representation of the nonlinear system by the NLDI model, and this feature is also one important contribution of this paper. Once the NLDI representation of the nonlinear system is obtained, the paper proposes the application of a linear control design method to this representation. The design is based on quadratic Lyapunov functions and formulated as search problem over a set of bilinear matrix inequalities (BMIs), which is solved using a two-step separation procedure that maps the BMIs into a set of corresponding linear matrix inequalities. Two numerical examples are given to demonstrate the effectiveness of the proposed approach.

KEYWORDS: nonlinear systems; linear dynamic output feedback control; linear differential inclusions; robust control; linear matrix inequalities.

\section{INTRODUCTION}

Stability analysis and control synthesis for complex systems (such as, nonlinear systems involving saturation or timevarying uncertainties) can be simplified by the use of linear descriptions of these dynamical systems in the form of linear differential inclusions (LDIs). Generally speaking, it may be possible to ensure that the trajectories of such a system exhibit certain features by analyzing a corresponding model in the form of an LDI. Necessary conditions that guarantee, for example, the existence of a polytopic LDI (PLDI) containing all trajectories of a particular nonlinear system are discussed and demonstrated in (Boyd et al., 1994; Hu and Chen, 2007).
A wide variety of analysis and control problems have been formulated and solved for different classes of LDIs, most of them in the form of linear matrix inequalities (LMIs) (e.g., (Boyd et al., 1994); (Xie and de Souza, 1992; Bernard et al., 1997)). The LMI approach makes it easy to include stability and performance specifications (such as, a minimum decay rate and bounds on the output peak values) in the formulation of analysis and synthesis problems for LDIs. Once the problem is written in terms of LMIs, efficient convex search or optimization methods can be used to find a solution to it (Boyd et al., 1994). Based on this feature, it may be easier to design a controller for a nonlinear system using a linear description of it in the form of an LDI.

The modeling, analysis and control of nonlinear systems via linear models are not restricted to approaches based on LDIs. In this sense, Mamdani and Takagi Sugeno fuzzy systems play also an important role (Montagner et al., 2010; Mozelli et al., 2010; Tognetti and Oliveira, 2010). These type of fuzzy systems allow nonlinear systems to be approximated by means of an averaged sum of linear models. Then, the problems of analysis and synthesis can be written in terms of LMIs (Montagner et al., 2010; Mozelli et al., 2010). Also, based on feedback linearization technique, adaptative neural networks or fuzzy control schemes have been introduced to approximate nonlinear systems into linear models (Chen et al., 1996).

This paper addresses both modeling and control of nonlinear systems via a particular class of LDIs: the class of the norm-bounded LDIs (NLDIs). The main idea behind the modelling technique is to represent the nonlinear system by a linear parameter-varying (LPV) system using the mean value theorem (Vidyasagar, 1993; Zemouche et al., 2005; Hossain et al., 2009). This LPV system can be particularly represented by a PLDI, provided that a certain set of conditions (that will be presented later in the paper) are satisfied.

It is possible to use LMIs to check the quadratic stability of a PLDI, but this requires the solution of one LMI for each of the vertices of the polytopic domain. One major drawback of this approach is the fact that, in general, the number of vertices of this polytope is high (see the numerical examples in (Hu and Chen, 2007; Hu, 2007)), and this translates into significant (and sometimes intractable) computational burden.

For some applications - especially the ones involving high dimensional problems (e.g. (Ramos et al., 2004; de Oliveira et al., 2009; Hossain et al., 2009)) - it may be easier to use a system description in the form of an NLDI, because checking its quadratic stability involve only two LMI constraints (Boyd et al., 1994). In this case, an efficient outer approximation (or overbounding) of a PLDI can be given by an NLDI (Boyd et al., 1994), which means that every trajectory of the 
former is also a trajectory of the latter. The formulation proposed in (Boyd et al., 1994) is based on the solution of an LMI feasibility problem and considers only the case where a specific matrix parameter of the NLDI is square and invertible. This condition is substituted here by a weaker one in which this matrix parameter must only have full column rank (and this is another important contribution of this paper). Using this weaker condition, it is possible to choose a particular structure of the NLDI parameters in order to reduce the conservatism in the representation of the underlying nonlinear system by the NLDI model.

Based on this approach for modeling a nonlinear system via an NLDI, this paper proposes the design of a linear dynamic output feedback (LDOF) controller for the NLDI model, in such a way that stability and performance specifications can be satisfied for the closed-loop nonlinear system using a linear controller. The motivation for focusing on this type of control comes from problems in the area of power systems stability (see, for example, (Basler and Schaefer, 2008; Ramos et al., 2004; Hossain et al., 2009)), in which linear controllers are often applied to a system with highly nonlinear behaviors, and these controllers must satisfy stability and performance specifications over a wide range of operating conditions. The control problem is formulated in this paper using quadratic Lyapunov functions and constraints in the form of bilinear matrix inequalities (BMIs).

Computational methods for solving BMIs are still under development and, in general, the existing ones are restricted to small dimensional problems (Polanski, 1997; Yfoulis and Shorten, 2004). However, it is possible to transform the particular set of BMIs resulting from the approach proposed in this paper into a set of LMIs, using a separation procedure presented in (de Oliveira et al., 2000). With this technique, it becomes possible to design a linear controller for the NLDI model of the original nonlinear system and, therefore, ensure that the closed-loop nonlinear system fulfills the stability and performance requirements within the whole operating region of interest.

This paper is organized as follows. Section 2 provides the problem formulation. Section 3 deals with the modeling problem, and this section is divided into five parts. The first part gives the basis for rewriting a nonlinear system as an LPV system; the second one defines a PLDI using the previously obtained LPV system; the third part presents a result that enables the overbounding of the PLDI by the NLDI model; the fourth part discusses the estimation of regions of attraction of nonlinear systems using NLDIs models and, the last part presents a numerical example that illustrate the application of the modeling procedure. Section 4 describes the fundamentals of the proposed controller design method, based on the NLDI model that was previously obtained. Sec- tion 6 presents some tests of the proposed control design procedure and its corresponding results. Finally, section 7 contains the conclusions and some final remarks on the proposed approach.

Notation: the notation used throughout this paper is standard. $\mathbb{R}^{n}$ denotes the $n$-dimensional Euclidean space, $\mathbb{R}^{n \times m}$ is the set of $n \times m$ real matrices. The closed convex polytope defined by a finite number of vertices, say $S_{1}, S_{2}, \ldots, S_{v}$, is defined as the convex hull of those elements and is denoted simply as $C o\left(S_{1}, S_{2}, \ldots, S_{v}\right)$. For two elements $a$ and $b$ in $\mathbb{R}^{n},\{a, b\}$ denotes the set constituted by only these two elements, while $[a, b]$ denotes the set containing all the points in the line segment between $a$ and $b$. For matrices and vectors ()$^{\prime}$ means transposition. For a symmetric matrix $P, P \succ 0$ $(P \prec 0)$ denotes positive (negative) definitess. Positive (negative) semi-definiteness is denoted by $P \succeq 0(P \preceq 0)$. An identity matrix with appropriate dimensions is denoted simply by $I$. For a matrix, $\|\cdot\|$ denotes the largest singular value of the matrix. For singular matrices, $(\cdot)^{+}$denotes the pseudoinverse of the matrix.

\section{PROBLEM FORMULATION}

Consider a continuous-time nonlinear system described in the state-space form by

$$
\dot{x}(t)=f(x(t))+B u(t), x(0)=x_{0},
$$

where $x(t)=\left[\begin{array}{lll}x_{1}(t) \ldots & x_{n}(t)\end{array}\right]^{\prime} \in X \subset \mathbb{R}^{n}$ is the state vector, $u(t) \in U \subset \mathbb{R}$ is the control input, $B$ is a constant matrix of proper dimension and $f: X \mapsto \mathbb{R}^{n}$ is a nonlinear function of class $C^{1}$. Here, the subset $X$ of $\mathbb{R}^{n}$ represents a state-space region of interest of (1) given by (Rohr et al., 2009)

$$
X:=\left\{x: a_{k} x \leq 1, k=1, \ldots, n_{e}\right\},
$$

where $a_{k} \in \mathbb{R}^{n}$ are given constant row vectors and $n_{e}$ is the number of edges of $X$.

This form of system (1), in which the nonlinearities are present only in the dynamics of the state with respect to itself, is a peculiarity of some practical systems (see (Ramos et al., 2004; de Oliveira et al., 2009)). For convinience, we assumed $x=0$ (with $u=0$ ) as being the equilibrium point of interest, so $f(0)=0,0 \in X$ and $0 \in U$. This assumption is quite standard and can be satisfied by a simple change of coordinates (Vidyasagar, 1993).

Initially, our goal is to model the nonlinear system (1) in the form of a linear differential inclusion defined as

$$
\dot{x}(t) \in D(x(t), u(t)), x(0)=x_{0},
$$

where $D(x(t), u(t)):=\{z(t) \quad: \quad z(t)=\Lambda x(t)+$ $B u(t), \forall \Lambda \in \Omega\}$, being $\Omega$ a non-empty, closed set of 
real $(n \times n)$-matrices (Boyd et al., 1994; Pyatnitskiy and Rapoport, 1996). Depending on the form of the set $\Omega$, different types of LDIs can be obtained. In this paper, we are interested in the class of norm-bounded LDIs, in which the set $\Omega$ has the particular form given by

$$
\Omega=\Omega_{N L D I}:=\left\{A_{0}+F E G:\|E\| \leq 1\right\},
$$

where $A_{0} \in \mathbb{R}^{n \times n}, F \in \mathbb{R}^{n \times n_{p}}$ and $G \in \mathbb{R}^{n_{q} \times n}$ are known fixed matrices, while $\mathrm{E}$ is any real $\left(n_{p} \times n_{q}\right)$-matrix satisfying $\|E\| \leq 1$. As is already well-known (Boyd et al., 1994), this NLDI (3)-(4) is equivalent to the linear time-varying system

$$
\dot{x}(t)=\left(A_{0}+\Delta A(t)\right) x(t)+B u(t), x(0)=x_{0},
$$

where $\Delta A(t)=F E(t) G$, being $E(t)$ an unknown matrix satisfying $\|E\| \leq 1$ for all $t>0$.

Consider again the nonlinear system (1). The mean value theorem guarantees the existence of a matrix $J(t)$ such that $f(x(t))=J(t) x(t)$, for every $x(t) \in X$. Hence, by an adequate choice of matrices $A_{0}, F$ and $G$ of (5), it can be possible to guarantee that $J(t) \in \Omega_{N L D I}$ for all $t>0$, which follows immediately that every trajectory of the nonlinear system (1) is also a trajectory of the NLDI (5).

In this paper, $A_{0}$ is assumed to be the Jacobian matrix obtained by truncating the Taylor series expansion of (1) (with $u=0$ ) at the first-order term, so the local properties of (1) are well described by (5). On the other hand, by a proper choice of matrices $F$ and $G$, the nonlinear behavior of (1) within the set $X$ is expected to be well described by the term $\Delta A(t)$. Section 3 discusses the main fundamentals of the proposed procedure to calculate these two matrices of the model.

Once we have a model of (1) in the form of the NLDI (5), the problem of interest is the determination of a linear dynamic output feedback controller in the state-space form

$$
\begin{aligned}
\dot{x_{c}}(t) & =A_{c} x_{c}(t)+B_{c} y(t), \\
u(t) & =C_{c} x_{c}(t),
\end{aligned}
$$

that stabilizes the system (5) and guarantee a desirable performance to the controlled system, where $x_{c}(t) \in \mathbb{R}^{n_{c}}$ and $y(t)=C x(t)$ is the measured output of (1). This will be discussed in section 4 .

\section{MODELING A NONLINEAR SYSTEM VIA NLDI}

The procedure proposed in this paper to calculate matrices $F$ and $G$ is based on the description of (1) in the form of an LPV system, within the state-space region of interest specified by $X$ in the previous section. This is done using the mean value theorem (Vidyasagar, 1993; Zemouche et al., 2005), as it is discussed in section 3.1. Using this reformulation of (1), section 3.2 shows that it is possible to define a politopic LDI whose set of trajectories contains all the solutions of the LPV system. Finally, in section 3.3 we calculate the matrices $F$ and $G$ by solving an optimization problem in the form of LMIs which guarantees that $\Omega_{N L D I} \supseteq \Omega_{P L D I}$, where $\Omega_{P L D I}$ is the particular form of $\Omega$ associated to the politopic LDI (Boyd et al., 1994; Pyatnitskiy and Rapoport, 1996).

\subsection{Rewriting the nonlinear system as an LPV system}

We present the version of the mean value theorem that is applicable to the general case where $f(x)=\left[f_{1}(x) \ldots f_{q}(x)\right]^{\prime}$, with $f_{i}: \mathbb{R}^{n} \mapsto \mathbb{R}, i=1, \ldots, q$. Consider the canonical basis of the vectorial space $\mathbb{R}^{s}$, for $s \geq 1$, given by $E_{s}=\left\{e_{s}(i): e_{s}(i)=(0, \ldots, 0,1,0, \ldots, 0)^{\prime}, i=1, \ldots, s\right\}$. Using the canonical basis $E_{q}$ of the vectorial space $\mathbb{R}^{q}$, it is possible to write $f(x)$ as $f(x)=\sum_{i=1}^{q} e_{q}(i) f_{i}(x)$. Now, we can state the following proposition (Zemouche et al., 2005).

Proposition 1 Let $f(x): \mathbb{R}^{n} \mapsto \mathbb{R}^{q}$. Let $a$ and $b$ two elements in $\mathbb{R}^{n}$, and assume that $f$ is differentiable on $C o(a, b)$. Then, there are constant vectors $c_{i} \in \operatorname{Co}(a, b), c_{i} \neq a, c_{i} \neq b, i=$ $1, \ldots, q$, such that

$f(a)-f(b)=\left(\sum_{i=1}^{q} \sum_{j=1}^{n} e_{q}(i) e_{n}^{\prime}(j) \frac{\partial f_{i}}{\partial x_{j}}\left(c_{i}\right)\right)(a-b)$.

For the proof, see (Zemouche et al., 2005). In this paper, we use this version of the mean value theorem to rewrite the nonlinear system (1) as an LPV system. We first assume that $f$ is differentiable on the line segment between $x(t)$ and the equilibrium point at the origin $x=0$, i.e., on the set $C o(x(t), 0)=\{\lambda x(t): \lambda \in[0,1]\}, \forall t>0$. Hence, Proposition 1 guarantees the existence of $n$ vectors $x_{s_{i}}(t) \in$ $C o(x(t), 0), x_{s i}(t) \neq x(t), x_{s_{i}}(t) \neq 0, i=1, \ldots, n$, such that

$$
f(x(t))=\left(\sum_{i=1}^{n} \sum_{j=1}^{n} Q_{i j} \frac{\partial f_{i}}{\partial x_{j}}\left(x_{s_{i}}(t)\right)\right) x(t), \forall t>0
$$

where $Q_{i j}=e_{n}(i) e_{n}(j)^{\prime}$. Now, let us define the functions

$$
h_{i j}(t)=\frac{\partial f_{i}}{\partial x_{j}}\left(x_{s_{i}}(t)\right)-\frac{\partial f_{i}}{\partial x_{j}}(0),
$$

where $i, j=1, \ldots, n$ and the second term in the right side of the equality (9) is the element $i \times j$ of the Jacobian matrix obtained by truncating the Taylor series expansion of (1) at the first-order term. This Jacobian matrix will be denoted simply by matrix $A_{0}$ and it describes the linear properties of 
(1) in the neighbourhood of the origin. So, we can write (8) as being

$$
f(x(t))=\left(A_{0}+\sum_{i=1}^{n} \sum_{j=1}^{n} Q_{i j} h_{i j}(t)\right) x(t) .
$$

Observe that the second term on right side of the equality (10) captures only the nonlinear behavior of (1). It is important to point out that the mean value theorem guarantees the existence of the vectors $x_{s_{i}}(t), i=1, \ldots, n$, for which the equality (10) is satisfied, but it does not provide any means to calculate them. We can deal with this problem, however, by setting a bounded range for the functions $h_{i j}(t), i, j=1, \ldots, n$. To do so, let us assume that,

$$
\sup _{t>0}\left|h_{i j}(t)\right|<\infty, i, j=1, \ldots, n .
$$

This allows us to specify a lower bound $\underline{h}_{i j}$ and an upper bound $\bar{h}_{i j}$ by

$$
\underline{h}_{i j}=\inf _{t>0} h_{i j}(t), \bar{h}_{i j}=\sup _{t>0} h_{i j}(t) .
$$

We can use the fact that the functions $h_{i j}$ are bounded (and their bounds are given by (12)) to obtain a description of the nonlinear system (1) within the region $X$ via the following LPV system with bounded parameters

$$
\dot{x}(t)=\left(A_{0}+\sum_{i=1}^{n} \sum_{j=1}^{n} Q_{i j} \theta_{i j}(t)\right) x(t)+B u(t),
$$

where $\theta_{i j}: \Re_{+} \mapsto\left[\underline{h}_{i j}, \bar{h}_{i j}\right], i, j=1, \ldots, n$ and $x(0)=x_{0}$.

Observe that the LPV system (13) captures the effects of the nonlinearities of (1) on the system dynamics by the time varying-parameters $\theta_{i j}(t), i, j=1, \ldots, n$. It is important to emphasize, however, that this description of (1) may be quite conservative, since the exact relationship between $\theta_{i j}(t)$ and $h_{i j}(t)$ (given by (9)) is neglected in (13). As a consequence, many trajectories of the LPV system (13) may not be trajectories of (1). This is the price we pay for representing the nonlinear system (1) in a linear form described by (13).

In relation to the practical meaning of the assumption (11) and the set $X$, notice that most of the physical systems operate within a bounded range of their variables (such as voltages, frequencies and rotor angles, for an electric power system (Basler and Schaefer, 2008; Ramos et al., 2004; Hossain et al., 2009)), which means that it is generally possible to set lower and upper bounds to the states of (1). These bounds will define a certain state-space region of interest $X \subset \mathbb{R}^{n}$ around the equilibrium point of interest. Hence, this set $X$ contains all the practical values of the state variables. Using these pre-specified bounds for the states, it is possible then, by an analysis of the mathematical expressions of (9), to define the upper and lower bounds to these functions $h_{i j}(t)$, $i, j=1, \ldots, n$. For some cases (when, for example, the functions $h_{i j}(t), i, j=1, \ldots, n$ are written as combinations of sine and cosine functions), however, it may be possible to ensure the assumption (11) for the entire state-space region $\mathbb{R}^{n}$. In these cases, global stabilization of the nonlinear system (1) can be evaluated via the NLDI model, but this possibility is not investigated in details in this paper.

Comparing the LPV system (13) to the NLDI in the form of (5), it becomes clear now that the proposal of this paper is to model the term $\sum_{i=1}^{n} \sum_{j=1}^{n} Q_{i j} \theta_{i j}(t)$ by the term $\Delta A(t)$ of (5), which amounts to choosing adequately the matrices $F$ and $G$. For that, notice that the LPV system (13) can be particularly represented in a polytopic form when $\left(A_{0}+\sum_{i=1}^{n} \sum_{j=1}^{n} Q_{i j} \theta_{i j}(t)\right)$ involves a polytopic domain of vertices. This particular form of (13) can be interpreted as a polytopic LDI, where all trajectories of the LPV system (13) will also be trajectories of this PLDI. This is discussed in the next section.

\subsection{Specifying a PLDI containing all the trajectories of the LPV system}

In this section, we are interested to model the LPV system (13) as a PLDI model defined in the form of the following linear time-varying system (Pyatnitskiy and Rapoport, 1996):

$$
\dot{x}(t)=\sum_{i=1}^{v} \alpha_{i}(t) S_{i} x(t)+B u(t), x(0)=x_{0},
$$

where the elements of the vector function $\alpha(t)=$ $\left[\alpha_{1}(t) \ldots \alpha_{v}(t)\right]^{\prime}$ satisfy the conditions

$$
0 \leq \alpha_{i}(t) \leq 1, \sum_{i=1}^{v} \alpha_{i}(t)=1, \forall t>0
$$

and $S_{i} \in \mathbb{R}^{n \times n}, i=1, \ldots, v$, is the $i^{t h}$ vertex of the convex set $\Omega_{P L D I}:=\operatorname{Co}\left(S_{1}, S_{2}, \ldots, S_{v}\right)$. So, we have $\sum_{i=1}^{v} \alpha_{i}(t) S_{i} \in \Omega_{P L D I}$ for every $\alpha(t)$ whose elements satisfy (15).

Considering the LPV system (13), it is possible to define a set $\Omega_{P L D I}$ such that $\left(A_{0}+\sum_{i=1}^{n} \sum_{j=1}^{n} Q_{i j} \theta_{i j}\right) \in \Omega_{P L D I}$, for all $\theta_{i j} \in\left[\underline{h}_{i j}, \bar{h}_{i j}\right], i, j=1, \ldots, n$, from which it follows immediately that every trajectory of (13) is also a trajectory of (14). For that, the set of the vertices of $\Omega_{P L D I}$ is defined by

$$
V_{S}=\left\{\left(A_{0}+\sum_{i=1}^{n} \sum_{j=1}^{n} Q_{i j} \theta_{i j}\right): \theta_{i j} \in\left\{\underline{h}_{i j}, \bar{h}_{i j}\right\}\right\} .
$$


The next step of the procedure consists in searching for a set $\Omega_{N L D I}$ that overbounds $\Omega_{P L D I}$. This is discussed in the next section.

\subsection{Overbounding the PLDI by the pro- posed NLDI model}

Our goal now is to find matrices $F$ and $G$ for (5) such that $\Omega_{N L D I} \supseteq \Omega_{P L D I}$, with the size of the set $\Omega_{N L D I}$ as small as possible. This will give an efficient outer approximation (or overbounding) of the PLDI (14) by the NLDI (5), from which it follows immediately that every trajectory of (14) is also a trajectory of (5) (Boyd et al., 1994). Reference (Boyd et al., 1994) formulates this problem in the form of LMIs. The result proposed in (Boyd et al., 1994) considers only the case with matrix $F$ square and nonsingular. Here, we relax this condition by a weaker one in which $F$ must only be full column rank.

Proposition 2 Let $\Omega_{N L D I}$ and $\Omega_{P L D I}$ be the sets defined for systems (5) and (14), respectively. We have $\Omega_{N L D I} \supseteq$ $\Omega_{P L D I}$ if there are a full column rank matrix $F$ and a matrix $G$ with proper dimension, such that

$$
\left[\begin{array}{cc}
G^{\prime} G & * \\
F^{+}\left(S_{i}-A_{0}\right) & I
\end{array}\right] \succeq 0, i=1, \ldots, v,
$$

where $A_{0}$ is a known matrix in $\Omega_{N L D I}$ and $S_{i}, i=1, \ldots, v$, are the vertices of $\Omega_{P L D I}$.

Proof We have $\Omega_{N L D I} \supseteq \Omega_{P L D I}$ if, for every $x(t)$ and $\alpha_{i}(t)$, $i=1, \ldots, v$, such that $0 \leq \alpha_{i}(t) \leq 1, \sum_{i=1}^{v} \alpha_{i}(t)=1$, there exists $E(t)$ satisfying

$$
\begin{gathered}
\left(A_{0}+F E(t) G\right) x(t)=\sum_{i=1}^{v} \alpha_{i}(t) S_{i} x(t), \\
x(t)^{\prime} G^{\prime} E(t)^{\prime} E(t) G x(t) \leq x(t)^{\prime} G^{\prime} G x(t),
\end{gathered}
$$

where $S_{i} \in V_{P L D I}$ is the $i^{t h}$ vertex of $\Omega_{P L D I}$. Notice that (19) is equivalent to the norm condition $\|E\| \leq 1$. We complete the proof by showing that (17) provides an equivalent expression of (18)-(19).

For that, it follows from (17) that

$$
\begin{aligned}
\sum_{i=1}^{v} \alpha_{i}(t)\left[\begin{array}{cc}
G^{\prime} G & * \\
F^{+}\left(S_{i}-A_{0}\right) & I
\end{array}\right] & \succeq 0 \Rightarrow \\
{\left[\begin{array}{cc}
\sum_{i=1}^{v} \alpha_{i}(t) G^{\prime} G & * \\
\sum_{i=1}^{v} \alpha_{i}(t) F^{+}\left(S_{i}-A_{0}\right) & \sum_{i=1}^{v} \alpha_{i}(t) I
\end{array}\right] } & \succeq 0 \Rightarrow \\
{\left[\begin{array}{cc}
F^{+}\left(\sum_{i=1}^{v} \alpha_{i}(t) S_{i}-A_{0}\right) & \text { I }
\end{array}\right] } & \succeq 0(20)
\end{aligned}
$$

for all $\alpha_{i}(t), i=1, \ldots, v$, satisfying $0 \leq \alpha_{i}(t) \leq 1$ and $\sum_{i=1}^{v} \alpha_{i}(t)=1$. Therefore, using Schur complements, the matrix inequality (20) is equivalent to

$$
\begin{array}{r}
\left(\sum_{i=1}^{v} \alpha_{i}(t) S_{i}-A_{0}\right)^{\prime} F^{+^{\prime}} F^{+}\left(\sum_{i=1}^{v} \alpha_{i}(t) S_{i}-A_{0}\right) \preceq G^{\prime} G \\
\Rightarrow \\
x(t)^{\prime}\left(\sum_{i=1}^{v} \alpha_{i}(t) S_{i}-A_{0}\right)^{\prime} F^{+^{\prime}} F^{+}\left(\sum_{i=1}^{v} \alpha_{i}(t) S_{i}-A_{0}\right) x(t) \leq \\
x(t) G^{\prime} G x(t)
\end{array}
$$

for all $x(t)$ and $\alpha_{i}(t), i=1, \ldots, v$, satisfying $0 \leq \alpha_{i}(t) \leq 1$ and $\sum_{i=1}^{v} \alpha_{i}(t)=1$. It can be concluded from this development that, if (17) is feasible for all $i=1, \ldots, v$, then the inequality (21) holds for all $x(t)$ and for all admissibles values of $\alpha_{i}(t), i=1, \ldots, v$.

Hence, to complete the proof we only need to show that (21) is equivalent to (18)-(19). This equivalence is easily verified by assuming that $F$ is a full column rank matrix. This assumption allows us to rewrite the equation (18) as $E(t) G x(t)=F^{+}\left(\sum_{i=1}^{v} \alpha_{i}(t) S_{i}-A_{0}\right) x(t)$. Now, the substitution of this expression into the inequality (19) leads exactly to (21), which completes the desired equivalence and the proof.

To solve the set of matrix inequalities (17) in the form of LMIs it is necessary to introduce the new variables

$$
V=G^{\prime} G, \quad W=F^{+},
$$

where $V \in \mathbb{R}^{n \times n}$ must be a symmetric and positive semidefinite matrix and $W \in \mathbb{R}^{n_{p} \times n}$. The condition of positive semidefinitess of matrix $V$ can be guaranteed by the additional constraint $V \succeq 0$. The condition on matrix $W$ can be dealt using LMI solvers specialized in rank constraints (Orsi et al., 2006), but this alternative is also not investigated in this paper. In this paper, the condition $\operatorname{rank}(W)=n_{p}$ is reinforced by imposing a particular, desired structure for $W$ and checked after the solution of the matrix inequalities (17) is obtained.

With the change of variables suggested by (22), our problem is to find matrices $V$ and $W$ such that

$$
\left[\begin{array}{cc}
V & * \\
W\left(S_{i}-A_{0}\right) & I
\end{array}\right] \succeq 0, i=1, \ldots, v,
$$

where matrices $A_{0}$ and $S_{i}, i=1, \ldots, v$, were already previously introduced.

Once we have calculated matrices $V$ and $W$ by solving the LMIs (23), matrix $F$ can be easily recovered as $F=W^{+}$. 
On the other hand, applying the Cholesky-like covariance decomposition to the matrix $V$ (and this is possible, once that $V$ is a positive semidefinite matrix), we find a matrix $G$ with $n_{q}=\operatorname{rank}(V)$. In order to obtain $\Omega_{N L D I} \supseteq \Omega_{P L D I}$, with the set $\Omega_{N L D I}$ as small as possible, reference (Boyd et al., 1994) proposes to solve the LMIs (23) as an optimization problem by minimizing the trace of matrix $V$. This alternative was adopted in this paper.

The weaker condition imposed on matrix $F$ (in which it must only be a full column rank matrix) allow us to adopt a particular structure to the matrix $F$ in such a way that the term $\Delta A(t)$ in (5) can better represent the nonlinear characteristics of (1). For example, if the $i^{\text {th }}$ equation of (1) is linear, it follows as a suggestion to set the elements of the $i^{\text {th }}$ row of $F$ to be equal to zero (which means that the $i^{t h}$ row of $\Delta A(t)$ will always be equal to zero, independently of the values of $E(t)$ ). To better clarify this point and the whole modeling procedure, consider the numerical example of the section 3.5. Before that, however, in section 3.4 the estimation of stability regions for the nonlinear system (1) using NLDIs is discussed.

\subsection{Estimation of regions of attractions for nonlinear systems using NLDIs}

In the sequence, we present a basic result from the Lyapunov theory that provides an estimate $D \subset X$ of the region of attraction of system (1) (Kiyama and Iwasaki, 2000; Coutinho and da Silva Jr., 2010).

Lemma 3 Consider the nonlinear system (1). Suppose there exist positive scalars $\epsilon_{1}, \epsilon_{2}$ and $\epsilon_{3}$ and a continuously differentiable function $V: X \mapsto \mathbb{R}$ that satisfies the following conditions:

$$
\begin{aligned}
& \epsilon_{1} x^{\prime} x \leq V(x) \leq \epsilon_{2} x^{\prime} x, \quad \forall x \in X \\
& \dot{V}(x) \leq-\epsilon_{3} x^{\prime} x, \quad \forall x \in X, \\
& D:=\{x: V(x) \leq 1\} \subset X .
\end{aligned}
$$

Then, $V(x)$ is a Lyapunov Function in $X$. Moreover, for all $x(0) \in D$, the trajectory $x(t)$ belongs to $D$ and approaches the origin as $t \rightarrow \infty$.

Next Lemma provides sufficient conditions to ensure that the region of attraction $D$ as defined by $D:=\left\{x: x^{\prime} P x \leq\right.$ 1, $\left.P=P^{\prime} \succ 0\right\}$ is bounded by the state-space region $X$ (Rohr et al., 2009).

Lemma 4 Consider the state-space region $X$ defined as (2). The condition $x \in X$ can be written as

$$
2-x^{\prime} a_{k}^{\prime}-a_{k} x \geq 0, \quad k=1, \ldots, n_{e} .
$$

Let the domain $D$ be defined as $D:=\left\{x: x^{\prime} P x \leq 1, P=\right.$ $\left.P^{\prime} \succ 0\right\}$. Thus, if $x \in D$, then

$$
x^{\prime} P x-1 \leq 0 .
$$

Thus, the condition $x \in D \subset X$ is guaranteed if the following inequality holds

$$
1-x^{\prime} a_{k}^{\prime}-a_{k} x+x^{\prime} P x \geq 0, \quad k=1, \ldots, n_{e} .
$$

Now, from Lemmas 3 and 4 and the ideas presented in (Rohr et al., 2009), it is possible to derive some sufficient conditions to ensure that $D$ with $V(x)=x^{\prime} P x, \quad P=P^{\prime} \succ 0$, is an estimate of the region of attraction of the nonlinear system (1) within the set $X$. It is important to emphasize that the calculation of this region $D$ is done for the representation of (1) in the form of the NLDI (5). This is allowed to do, once that the NLDI model obtained from the proposed modeling procedure represents the behavior of the nonlinear system (1) within the subset $X$.

Lemma 5 Consider the nonlinear system (1) and its representation in the form of the NLDI (5). Suppose there exist a matrix $P=P^{\prime} \succ 0$ and a scalar $\lambda>0$ satisfying the following LMIs:

$$
\begin{aligned}
& {\left[\begin{array}{rr}
A_{0}^{\prime} P+P A_{0}+\lambda G^{\prime} G & P F \\
* & -\lambda I
\end{array}\right] \prec 0,} \\
& {\left[\begin{array}{cc}
1 & a_{k} \\
* & P
\end{array}\right] \succeq 0, k=1, \ldots, n_{e}}
\end{aligned}
$$

Then, $V(x)=x^{\prime} P x$ is a Lyapunov Function in $X$. Moreover, for all $x(0) \in D$ the trajectory $x(t)$ approaches the origin when $t \rightarrow \infty$, where $D:=\{x: V(x) \leq 1\} \subset X$.

Proof Let $\epsilon_{1}$ and $\epsilon_{2}$ be, respectively, the smallest and largest eigenvalues of $P$. Then, the following inequalities hold for all $x \in X$

$$
\epsilon_{1} x^{\prime} x \leq x^{\prime} P x \leq \epsilon_{2} x^{\prime} x,
$$

which leads to the condition (24) of Lemma 3. Now, from (Boyd et al., 1994), it follows that if the LMI (30) is satisfied for a matrix $P=P^{\prime} \succ 0$ and a scalar $\lambda>0$, then we have $\dot{V}(x)<0$ for all $x \in X$, where $V(x)=x^{\prime} P x$. As $x$ is bounded, there exists a sufficiently small positive scalar $\epsilon_{3}$ such that

$$
\dot{V}(x) \leq-\epsilon_{3} x^{\prime} x,
$$

which leads to the condition (25) of Lemma 3. Now, we only have to prove that estimate $D:=\{x: V(x) \leq 1\}$ is bounded by $X$. To do so, pre- and post-multiply LMI (31) by $\left[\begin{array}{ll}1 & -x\end{array}\right]^{\prime}$ and its transpose. It provides

$$
\left[\begin{array}{c}
1 \\
-x
\end{array}\right]^{\prime}\left[\begin{array}{cc}
1 & a_{k} \\
* & P
\end{array}\right]\left[\begin{array}{c}
1 \\
-x
\end{array}\right] \succeq 0, k=1, \ldots, n_{e} .
$$

Notice that (34) is the matrix form of (29). This completes the proof. 
Lemma 5 provides an estimate $D \subset X$ of the region of attraction of the nonlinear system (1), using the representation of this system in the form of the NLDI (5). In order to find the largest estimate $D$ inside $X$, we may solve the LMIs in Lemma 5 as an optimization problem by minimizing the trace of matrix $P$, as suggested in (Rohr et al., 2009).

The application of the modeling procedure proposed in this section and the estimate of the system region of attraction are illustrated in the numerical example given in the next section.

\subsection{Numerical example 1}

Consider the following nonlinear system, where the state vector $x(t)$ is given by $x(t)=\left[\begin{array}{lll}u(t) & v(t) & z(t)\end{array}\right]^{\prime}$ (Topcu and Packard, 2009):

$$
\begin{aligned}
\dot{u}(t)= & f_{1}(x(t))=-3 u(t)-1.35 v(t)-0.56 z(t)+ \\
& 0.08 u(t) v(t)+0.44 v^{2}(t)+0.01 v(t) z(t)+ \\
& 0.22 v^{3}(t) \\
\dot{v}(t)= & f_{2}(x(t))=0.91 u(t)-0.64 v(t)-0.02 z(t)- \\
& 0.05 v^{2}(t)+0.11 v(t) z(t)-0.05 z^{2}(t) \\
\dot{z}(t)= & f_{3}(x(t))=u(t) .
\end{aligned}
$$

Notice that $f_{1}(x(t))$ and $f_{2}(x(t))$ are nonlinear functions of the state variables $u(t), v(t)$ and $z(t)$ and $f_{3}(x(t))$ is a linear function of the state $u(t)$. Let us consider the equilibrium point at the origin $x_{e}=\left[\begin{array}{lll}0 & 0 & 0\end{array}\right]^{\prime}$. The Jacobian matrix $A_{0}$ was calculated via Taylor series expansion of system (35)(37) around the equilibrium point $x_{e}$. The result of this process is given by

$$
\begin{aligned}
A_{0}= & {\left[\begin{array}{lll}
\frac{\partial f_{1}}{\partial u}(0) & \frac{\partial f_{1}}{\partial v}(0) & \frac{\partial f_{1}}{\partial z}(0) \\
\frac{\partial f_{2}}{\partial u}(0) & \frac{\partial f_{2}}{\partial v}(0) & \frac{\partial f_{2}}{\partial z}(0) \\
\frac{\partial f_{3}}{\partial u}(0) & \frac{\partial f_{3}}{\partial v}(0) & \frac{\partial f_{3}}{\partial z}(0)
\end{array}\right] } \\
= & {\left[\begin{array}{ccc}
-3 & -1.35 & -0.56 \\
0.91 & -0.64 & -0.02 \\
1 & 0 & 0
\end{array}\right] . }
\end{aligned}
$$

The first step of the modeling procedure is to rewrite the nonlinear system (35)-(37) in the form of an LPV system. For that, Proposition 1 of section 3.1 guarantees the existence of two vectors $x_{s_{1}}(t)=\left[\begin{array}{lll}u_{s_{1}}(t) & v_{s_{1}}(t) & z_{s_{1}}(t)\end{array}\right]^{\prime} \in C o(x(t), 0)$ and $x_{s_{2}}(t)=\left[\begin{array}{lll}u_{s_{2}}(t) & v_{s_{2}}(t) & z_{s_{2}}(t)\end{array}\right]^{\prime} \in C o(x(t), 0), x_{s_{i}} \neq$ $x(t), x_{s_{i}} \neq 0, i=1,2$, such that

$$
\begin{aligned}
& {\left[\begin{array}{l}
f_{1}(x(t)) \\
f_{2}(x(t)) \\
f_{3}(x(t))
\end{array}\right]=} \\
& =\left[\begin{array}{ccc}
\frac{\partial f_{1}}{\partial u}\left(x_{s_{1}}(t)\right) & \frac{\partial f_{1}}{\partial v}\left(x_{s_{1}}(t)\right) & \frac{\partial f_{1}}{\partial z}\left(x_{s_{1}}(t)\right) \\
\frac{\partial f_{2}}{\partial u}\left(x_{s_{2}}(t)\right) & \frac{\partial f_{2}}{\partial v}\left(x_{s_{2}}(t)\right) & \frac{\partial f_{2}}{\partial z}\left(x_{s_{2}}(t)\right) \\
1 & 0 & 0
\end{array}\right]\left[\begin{array}{l}
u(t) \\
v(t) \\
z(t)
\end{array}\right]
\end{aligned}
$$

for all $t>0$. Notice that, once the function $f_{3}(x(t))$ is linear, it is not altered by the application of the mean value theorem. As discussed in section 3.1, we can write this last equation as being

$$
\left[\begin{array}{l}
f_{1}(x(t)) \\
f_{2}(x(t)) \\
f_{3}(x(t))
\end{array}\right]=\left(A_{0}+\left[\begin{array}{ccc}
h_{11}(t) & h_{12}(t) & h_{13}(t) \\
h_{21}(t) & h_{22}(t) & h_{23}(t) \\
0 & 0 & 0
\end{array}\right]\right)\left[\begin{array}{l}
u(t) \\
v(t) \\
z(t)
\end{array}\right]
$$

where,

$$
\begin{aligned}
h_{11}(t) & =\frac{\partial f_{1}}{\partial u}\left(x_{s_{1}}(t)\right)-\frac{\partial f_{1}}{\partial u}(0), \\
h_{12}(t) & =\frac{\partial f_{1}}{\partial v}\left(x_{s_{1}}(t)\right)-\frac{\partial f_{1}}{\partial v}(0), \\
h_{13}(t) & =\frac{\partial f_{1}}{\partial z}\left(x_{s_{1}}(t)\right)-\frac{\partial f_{1}}{\partial z}(0), \\
h_{21}(t) & =\frac{\partial f_{2}}{\partial u}\left(x_{s_{2}}(t)\right)-\frac{\partial f_{2}}{\partial u}(0), \\
h_{22}(t) & =\frac{\partial f_{2}}{\partial v}\left(x_{s_{2}}(t)\right)-\frac{\partial f_{2}}{\partial v}(0), \\
h_{23}(t) & =\frac{\partial f_{2}}{\partial z}\left(x_{s_{2}}(t)\right)-\frac{\partial f_{2}}{\partial z}(0),
\end{aligned}
$$

Calculating the functions $h_{i j}(t)$ (for $i=1,2$ and $j=1,2,3$ ) from (38)-(43), we have

$$
\begin{aligned}
h_{11}(t)= & 0.08 v_{s_{1}}(t) \\
h_{12}(t)= & 0.08 u_{s_{1}}(t)+0.88 v_{s_{1}}(t)+0.01 z_{s_{1}}(t)+ \\
& +0.66 v_{s_{1}}^{2}(t) \\
h_{13}(t)= & 0.01 v_{s_{1}}(t) \\
h_{21}(t)= & 0 \\
h_{22}(t)= & -0.1 v_{s_{2}}(t)+0.11 z_{s_{2}}(t) \\
h_{23}(t)= & 0.11 v_{s_{2}}(t)-0.1 z_{s_{2}}(t)
\end{aligned}
$$

The bounds $\underline{h}_{i j}$ and $\bar{h}_{i j}$ as defined by (12) were calculated by analysing the mathematical expressions of the functions (44)-(49) considering the following state-space region

$$
\begin{aligned}
X:= & \left\{\left[\begin{array}{lll}
u & v & z
\end{array}\right]^{T} \in \mathbb{R}^{3} \mid-1 \leq u \leq 1,-\frac{\pi}{2} \leq v \leq \frac{\pi}{2},\right. \\
& \left.-\frac{\pi}{2} \leq z \leq \frac{\pi}{2}\right\}
\end{aligned}
$$

This state-space region was specified by assuming it as being an operation region of the system or the region containing the practical values of the states. Once we have delimited the state-space region of interest, the points $x_{s_{i}}(t), i=1,2$, are also bounded, once that $x_{s_{i}}(t) \in C o(x(t), 0)$, for all $t>0$. So, by analysing the mathematical expressions of the functions (44)-(49) it was possible to define the following upper and lower bounds: $\underline{h}_{11}=-0.126, \bar{h}_{11}=0.126, \underline{h}_{12}=0.150$, 
$\bar{h}_{12}=3.106, \underline{h}_{13}=-0.015, \bar{h}_{13}=0.015, \underline{h}_{22}=-0.329$, $\bar{h}_{22}=0.329, \underline{h}_{23}=-0.329, \bar{h}_{23}=0.329$.

To better understand the calculation of these bounds, let us take $h_{11}(t)$ as an example. It is function of the point $v_{s_{1}}(t)$, whose value must be in the line segment between $-\frac{\pi}{2}$ and $\frac{\pi}{2}$, for all $t>0$. Hence, the maximum value that $h_{11}(t)$ can achieve (i.e, the upper bound $\bar{h}_{11}$ ) is 0.126 and the smallest value (i.e, the lower bound $\underline{h}_{11}$ ) is -0.126 .

With the specified bounds to the functions (44)-(49), we have a description of the nonlinear system (35)-(37) in the form of the following LPV system

$$
\begin{aligned}
& {\left[\begin{array}{l}
\dot{x}_{1}(t) \\
\dot{x}_{2}(t) \\
\dot{x}_{3}(t)
\end{array}\right]=} \\
& =\left(A_{0}+\left[\begin{array}{ccc}
\theta_{11}(t) & \theta_{12}(t) & \theta_{13}(t) \\
0 & \theta_{22}(t) & \theta_{23}(t) \\
0 & 0 & 0
\end{array}\right]\right)\left[\begin{array}{l}
u(t) \\
v(t) \\
z(t)
\end{array}\right],
\end{aligned}
$$

where $\theta_{i j}: \Re_{+} \rightarrow\left[\underline{h}_{i j}, \bar{h}_{i j}\right], i=1,2, j=1,2,3(j \neq 1$ when $i=2$ ).

The second step of the modeling procedure consists of specifying a PLDI in the form of (14) containing all the trajectories of the LPV system (51). For that, we only need to construct a set $\Omega_{P L D I}$ as defined by (16). Once that the LPV system (51) has 5 non-zero functions $\theta_{i j}(t)$, so the corresponding set $\Omega_{P L D I}$ has 32 vertices.

To complete the modeling procedure, we have to overbound the PLDI by the proposed NLDI model. This is done by solving the LMI problem introduced in section 3.3. The two matrices of the model that we must calculate are $G$ and $F$. The standard method proposed in (Boyd et al., 1994) requires that $F$ be a square and nonsingular matrix so, in this case, we should have to calculate a full rank $3 \times 3$ matrix. As a result, the third line of the term $\Delta A(t)$ of the NLDI model can have non-zero elements. But, notice that $f_{3}(x(t))$ of our example is linear, so the third line of $\Delta A(t)$ is expected to be equal to zero (which means that all the elements of this line are equal to zero) to avoid conservatism.

On the other hand, by applying the relaxing condition on matrix $F$, as proposed in Proposition 2 of section 3.3, we can set a matrix $F$ in the form $F=\left[\begin{array}{lll}f_{11} & \mathrm{f}_{21} & 0\end{array}\right]^{\prime}$. In this case, the row with the element equal to zero (row 3 ) forces the respective row of the matrix $\Delta A(t)$ to also have all its elements equal to zero, independently of any value of $E(t)$ and $G$. Hence, the obtained NLDI model will mantain the characteristic of the study system with respect to the fact that $f_{3}(x(t))$ is linear. Another possible advantage of Proposition 2 (in comparison to the standard method proposed in (Boyd et al., 1994)) is the fact that the structure of the matrix $F$ can be choosen in or- der to reduce the number of elements to be determined by the LMI optimization problem. To better clarify this point, notice that matrix $F$ has 9 elements to be calculated by applying the standard method, while the proposed method allows us to specify a matrix $F$ with only 2 elements to be calculated.

For the matrix $G$, we set it as a $3 \times 3$ matrix, so the matrix variable $V$ was choosen to be a $3 \times 3$ symmetric matrix. In addition, we imposed the following structure to the matrix variable $W$ in order to obtain a matrix $F$ in the form of $F=$ $\left[\begin{array}{lll}f_{11} & f_{21} & 0\end{array}\right]^{\prime}$ :

$$
W=\left[\begin{array}{lll}
\mathrm{w}_{11} & \mathrm{w}_{12} & 0
\end{array}\right]
$$

From the solution of the optimization problem suggested in section 3.3 using the SeDuMi solver (Sturm, 1999) in conjunction with YALMIP (Lofberg, 2004) it was obtained the following matrices $F$ and $G$

$$
F=\left[\begin{array}{c}
0.0663 \\
0.0044 \\
0
\end{array}\right], G=\left[\begin{array}{ccc}
6.0181 & 0 & 0 \\
0 & 17.953 & 0 \\
0 & 0 & 2.9495
\end{array}\right]
$$

This complete the modeling procedure. Now, let us estimate the region of attraction of the nonlinear system (35)-(37) with respect to the origin by using its representation in the form of the NLDI model previously calculated. This was done using Lemma 5. In order to obtain a solution for the LMI (31), we have to describe the set $X$ in the form given by (2). This was done by following the ideas presented in (Rohr et al., 2009). At first, notice that (50) is equivalent to a polytope $X \subset \mathbb{R}^{3}$ whose vertices are defined by $\Theta:=\left\{c_{1}, c_{2}, \ldots, c_{8}\right\}$, where

$$
\begin{aligned}
& c_{1}=\left[\begin{array}{c}
1 \\
-\pi / 2 \\
-\pi / 2
\end{array}\right], c_{2}=\left[\begin{array}{c}
1 \\
-\pi / 2 \\
\pi / 2
\end{array}\right], c_{3}=\left[\begin{array}{c}
-1 \\
-\pi / 2 \\
\pi / 2
\end{array}\right], \\
& c_{4}=\left[\begin{array}{c}
-1 \\
-\pi / 2 \\
-\pi / 2
\end{array}\right], c_{5}=\left[\begin{array}{c}
-1 \\
\pi / 2 \\
-\pi / 2
\end{array}\right], c_{6}=\left[\begin{array}{c}
-1 \\
\pi / 2 \\
\pi / 2
\end{array}\right], \\
& c_{7}=\left[\begin{array}{c}
1 \\
\pi / 2 \\
\pi / 2
\end{array}\right], c_{8}=\left[\begin{array}{c}
1 \\
\pi / 2 \\
-\pi / 2
\end{array}\right] .
\end{aligned}
$$

A vertex representation of $X$ is defined as the convex hull of $c_{1}, c_{2}, \ldots, c_{8}$, i.e., $X=C o\left(c_{1}, c_{2}, \ldots, c_{8}\right)$. Equivalently, we can define this vertex form of $X$ as (2):

$$
X:=\left\{x: a_{k} x \leq 1, \quad k=1, \ldots, 12\right\} .
$$

In order to calculate the vectors $a_{k} \in \mathbb{R}^{3}$, notice that each inequality defines an hyperplane $\left\{x: a_{k} x \leq 1\right\}$ for which 
two vertices belongs to its edge, that is

$$
\begin{array}{ll}
c_{1}, c_{2} \in\left\{x: a_{1} x=1\right\}, & c_{2}, c_{3} \in\left\{x: a_{2} x=1\right\}, \\
c_{3}, c_{4} \in\left\{x: a_{3} x=1\right\}, & c_{1}, c_{4} \in\left\{x: a_{4} x=1\right\}, \\
c_{4}, c_{5} \in\left\{x: a_{5} x=1\right\}, & c_{5}, c_{6} \in\left\{x: a_{6} x=1\right\}, \\
c_{6}, c_{7} \in\left\{x: a_{7} x=1\right\}, & c_{7}, c_{8} \in\left\{x: a_{8} x=1\right\}, \\
c_{5}, c_{8} \in\left\{x: a_{9} x=1\right\}, & c_{1}, c_{8} \in\left\{x: a_{10} x=1\right\}, \\
c_{2}, c_{7} \in\left\{x: a_{11} x=1\right\}, & c_{3}, c_{6} \in\left\{x: a_{12} x=1\right\} .
\end{array}
$$

Figure 1 shows the set $X$ with its vertices and illustration of some of its edges $\left(\left\{x: a_{1} x=1\right\},\left\{x: a_{7} x=1\right\}\right.$ and $\left.\left\{x: a_{11} x=1\right\}\right)$.

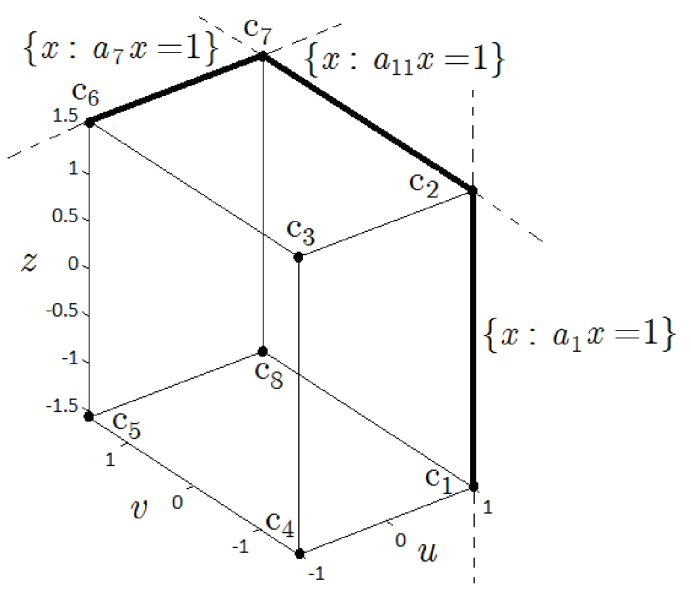

Figure 1: Region $X$ with its vertices $c_{1}, \ldots, c_{8}$ and illustration of some of its edges $\left(\left\{x: a_{1} x=1\right\},\left\{x: a_{7} x=1\right\}\right.$ and $\left.\left\{x: a_{11} x=1\right\}\right)$.

For a given set $\Theta$, the row vectors $a_{k}$ can be determined by solving the following set of linear systems:

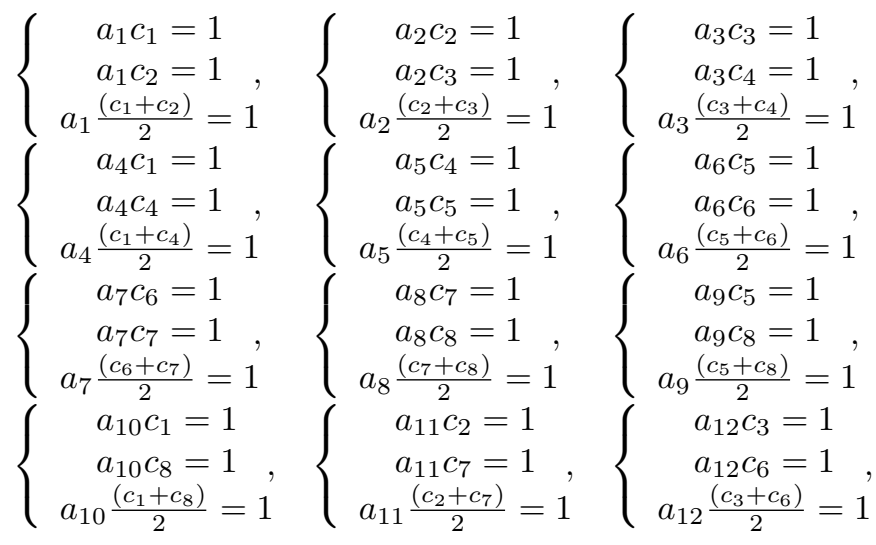

yielding the following row vectors

$$
\begin{aligned}
& a_{1}=\left[\begin{array}{lll}
0.29 & -0.45 & 0
\end{array}\right], a_{2}=\left[\begin{array}{lll}
0 & -0.32 & 0.32
\end{array}\right], \\
& a_{3}=\left[\begin{array}{lll}
-0.29 & -0.45 & 0
\end{array}\right], a_{4}=\left[\begin{array}{lll}
0 & -0.32 & -0.32
\end{array}\right], \\
& a_{5}=\left[\begin{array}{lll}
-0.29 & 0 & -0.45
\end{array}\right], a_{6}=\left[\begin{array}{lll}
-0.29 & 0.45 & 0
\end{array}\right], \\
& a_{7}=\left[\begin{array}{lll}
0 & 0.32 & 0.32
\end{array}\right], a_{8}=\left[\begin{array}{lll}
0.29 & 0.45 & 0
\end{array}\right], \\
& a_{9}=\left[\begin{array}{lll}
0 & 0.32 & -0.32
\end{array}\right], a_{10}=\left[\begin{array}{lll}
0.29 & 0 & -0.45
\end{array}\right] \text {, } \\
& a_{11}=\left[\begin{array}{lll}
0.29 & 0 & 0.45
\end{array}\right], a_{12}=\left[\begin{array}{lll}
-0.29 & 0 & 0.45
\end{array}\right] \text {. }
\end{aligned}
$$

The ellipsoid $D:=\left\{x \in \mathbb{R}^{3} \mid x^{\prime} P x \leq 1\right\}$ was used for the estimation of the region of attraction of the nonlinear system. To calculate the largest estimate in region $X$, the LMIs of Lemma 5 were solved as an optimization problem by minimizing the trace of matrix $P$. The calculated matrix $P$ is

$$
P=\left[\begin{array}{ccc}
0.307 & -0.001 & 0.013 \\
* & 0.285 & -0.031 \\
* & * & 0.301
\end{array}\right]
$$

Figure 2 shows the estimated region of attraction $D$ and the state-space region $X$ defined as (50). It is important to emphasize that $D$ is an estimate of the region of attraction of the nonlinear system (35)-(37) with respect to the origin. It was calculated via Lemma 5 by using the representation of the nonlinear system in the form of the NLDI previously calculated.

Next section presents the theory towards the design technique of the dynamic output feedback controller.

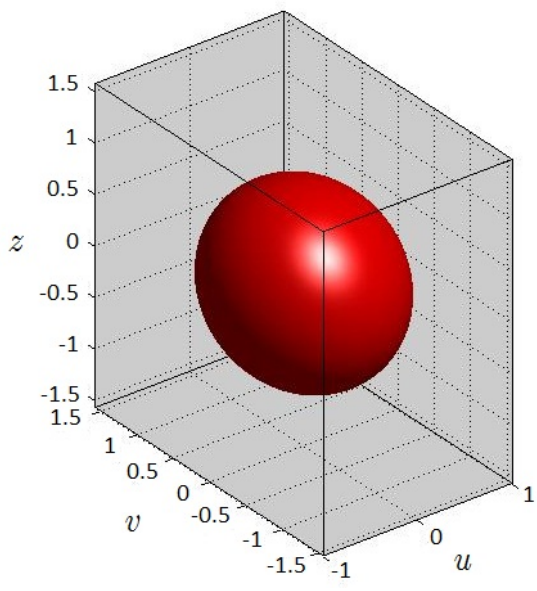

Figure 2: Region $X$ (the box bounded by the faces in gray); region of attraction $D$ of the origin of the nonlinear system (in red). 


\section{DYNAMIC OUTPUT FEEDBACK CON- TROLLER DESIGN}

Let the controlled system formed by the interconnection between (5) and (6)-(7) (with $y(t)=C x(t)$ ) be described as

$$
\dot{\tilde{x}}(t)=\left(\tilde{A}_{0}+\Delta \tilde{A}(t)\right) \tilde{x}(t)
$$

where $\Delta \tilde{A}(t)=\tilde{F} E(t) \tilde{G}$, with $E(t)$ satisfying $\|E(t)\| \leq 1$, for all $t>0$. Besides, $\tilde{x}(t)=\left[x(t) \quad x_{c}(t)\right]^{\prime}$, and

$$
\tilde{A}_{0}=\left[\begin{array}{cc}
A_{0} & B C_{c} \\
B_{c} C & A_{c}
\end{array}\right], \tilde{F}=\left[\begin{array}{c}
F \\
0
\end{array}\right], \tilde{G}=\left[\begin{array}{c}
G^{\prime} \\
0
\end{array}\right]^{\prime}
$$

In many applications, it is desirable that the system trajectories approach the origin as fast as possible (Folcher and Ghaoui, 1994; Silva and Junior, 2006). This practical requirement can be fulfilled by guaranteeing that $\lim _{t \rightarrow \infty} e^{\alpha t} \|$ $x(t) \|=0$ for all trajectories of (55), where $\alpha$ is defined as being the decay rate of (55). This can be done by satisfying the condition $\dot{V}(\tilde{x}(t)) \leq-2 \alpha V(\tilde{x}(t))$ (Boyd et al., 1994), where $V(\tilde{x}(t))=\tilde{x}(t)^{\prime} \tilde{P} \tilde{x}(t)$ is the Lyapunov function adopted to the NLDI model. This condition is equivalent to the existence of a positive definite symmetric matrix $\tilde{P} \in$ $\mathbb{R}^{\left(n+n_{c}\right) \times\left(n+n_{c}\right)}$ and matrices $A_{c}, B_{c}$ and $C_{c}$ such that

$$
\left[\begin{array}{cc}
\tilde{A}_{0}^{\prime} \tilde{P}+\tilde{P} \tilde{A}_{0}+\tilde{G}^{\prime} \tilde{G}+2 \alpha \tilde{P} & \tilde{P} \tilde{F} \\
* & -I
\end{array}\right] \prec 0 .
$$

In addition, it may be desirable that all modes of response (eigenvalues) of the closed-loop matrix $\tilde{A}_{0}$ have damping ratios larger than a minimum, pre-defined value (see the applications in (Ramos et al., 2004; de Oliveira et al., 2009)). To comply with this, we impose an additional restriction on the problem formulation using the regional pole placement (RPP) technique (Chiali et al., 1999). This technique consists in the definition of a region for pole placement in the complex plane where the design objective is fulfilled. This region is defined by all the complex numbers that have a damping ratio $\xi$ higher (or equal) than $\xi_{m i n}$, and it can be viewed in Figure 3 , where $\xi_{\text {min }}$ is the desired minimum damping ratio for the eigenvalues of matrix $\tilde{A}_{0}$ and $\delta=\cos ^{-1} \xi_{\text {min }}$. Note that the damping ratio is a local property, which only makes sense when the trajectory is close enough to the equilibrium point of interest.

All the eigenvalues of matrix $\tilde{A}_{0}$ are located within the region specified in Figure 3 if there exists a positive definite symmetric matrix $\tilde{P}$ and matrices $A_{c}, B_{c}$ and $C_{c}$ such that (Chiali et al., 1999)

$$
\left[\begin{array}{cc}
\left(\tilde{A}_{0}^{\prime} \tilde{P}+\tilde{P} \tilde{A}_{0}\right) \sin (\delta) & \left(\tilde{P} \tilde{A}_{0}-\tilde{A}_{0}^{T} \tilde{P}\right) \cos (\delta) \\
* & \left(\tilde{A}_{0}^{\prime} \tilde{P}+\tilde{P} \tilde{A}_{0}\right) \sin (\delta)
\end{array}\right] \prec 0 .
$$

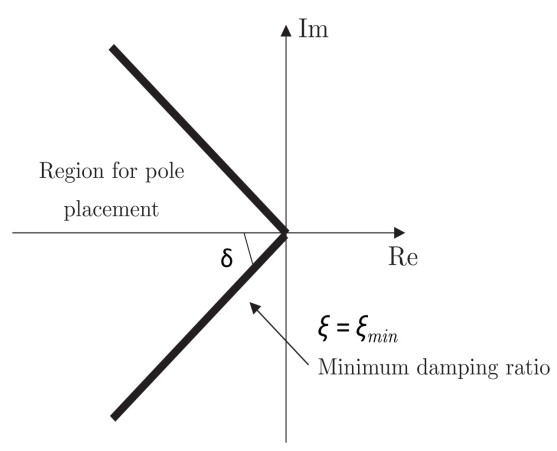

Figure 3: Region for pole placement.

\subsection{Robustness of the controller with re- spect to the variations in the operat- ing conditions of the system}

To deal with the robustness of the controller with respect to the different operating points of the system, we obtain a description of the system (1) in a certain state-space region around each equilibrium point of interest. The idea is to design a fixed parameter controller in the form (6)-(7) that exhibits an effective performance in all of these regions. This controller, however, must not change the equilibrium points of the open-loop sytem. In fact, once that a certain initial condition is within the region of attraction of a particular equilibrium (say, $x_{e 1}$ ), then the control objective is to force the system trajectories to approach the point $x_{e 1}$ as fast as possible, in accordance to the practical requirements discussed in the previous section.

The set of resulting controlled systems are described in state space form by

$$
\dot{\tilde{x}}^{(i)}(t)=\left(\tilde{A}_{0 i}+\Delta \tilde{A}_{i}(t)\right) \tilde{x}^{(i)}(t), \quad i=1, \ldots, n_{p},
$$

where $\Delta \tilde{A}_{i}(t)=\tilde{F}_{i} E_{i}(t) \tilde{G}_{i}$, with $E_{i}(t)$ satisfying $\|$ $E_{i}(t) \| \leq 1$, for all $t>0$. We also have

$$
\begin{aligned}
& \tilde{A}_{0 i}=\left[\begin{array}{cc}
A_{0 i} & B_{i} C_{c} \\
B_{c} C_{i} & A_{c}
\end{array}\right], \tilde{F}_{i}=\left[\begin{array}{c}
F_{i} \\
0
\end{array}\right], \tilde{G}_{i}=\left[\begin{array}{c}
G_{i}^{\prime} \\
0
\end{array}\right]^{\prime}, \\
& \tilde{x}^{(i)}(t)=\left[\begin{array}{c}
x(t)-x_{e i} \\
x_{c}(t)
\end{array}\right],
\end{aligned}
$$

being $x_{e i}$ the $i^{t h}$ equilibrium point of interest of (1).

\subsection{The complete control problem}

Grouping the constraints (56)-(57) with the robusteness requirement shown in previous subsection, it is possible to formulate the complete control problem as a search for positive 
definite symmetric matrices $\tilde{P}_{i}, i=1, \ldots, n_{p}$, and matrices $A_{c}, B_{c}$ and $C_{c}$ of proper dimensions such that

$$
\begin{gathered}
{\left[\begin{array}{cc}
\tilde{A}_{0 i}^{\prime} \tilde{P}_{i}+\tilde{P}_{i} \tilde{A}_{0 i}+\tilde{G}_{i}^{T} \tilde{G}_{i}+2 \alpha \tilde{P}_{i} & \tilde{P}_{i} \tilde{F}_{i} \\
& -I
\end{array}\right] \prec 0, \quad \text { (59) }} \\
{\left[\begin{array}{cc}
\left(\tilde{A}_{0 i}^{\prime} \tilde{P}_{i}+\tilde{P}_{i} \tilde{A}_{0 i}\right) \sin (\delta) & \left(\tilde{P}_{i} \tilde{A}_{0 i}-\tilde{A}_{0 i}^{T} \tilde{P}_{i}\right) \cos (\delta) \\
* & \left(\tilde{A}_{0 i}^{\prime} \tilde{P}_{i}+\tilde{P}_{i} \tilde{A}_{0 i}\right) \sin (\delta)
\end{array}\right] \prec 0 .}
\end{gathered}
$$

Notice that (59)-(60) are bilinear matrix inequalities (BMIs), since there are cross-products among the controller variables (i.e., $A_{c}, B_{c}$ and $C_{c}$ ) and matrices $\tilde{P}_{i}, i=1, \ldots, n_{p}$. In this paper, we apply a two-step separation procedure that allows to transform the BMIs (59)-(60) into a set of LMIs (de Oliveira et al., 2000). Basically, this separation procedure consists on the parameterization of some matrix variables and the definition of some new variables.

\section{SOLVING THE BMI PROBLEM: THE TWO-STEP SEPARATION PROCEDURE}

The concepts and procedures described in this section are derived from the ideas presented in (de Oliveira et al., 2000). Consider the set of BMIs (59)-(60). Let us assume that: $(i)$ $\tilde{P}=\tilde{P}_{i}, i=1, \ldots, n_{p} ;$ (ii) the dimension of the controller is equal to the dimension of the plant to be controlled, i.e., $n_{c}=n$, and; (iii) the controller output matrix $C_{c}$ is previously known. Let us partionate the matrices $\tilde{P}$ and its inverse $\tilde{P}^{-1}$ and define a matrix $\tilde{T}$ as follows

$\tilde{P}=\left[\begin{array}{cc}X & U \\ * & X_{c}\end{array}\right], \tilde{P}^{-1}=\left[\begin{array}{cc}Y & Y \\ * & Y_{c}\end{array}\right], \tilde{T}=\left[\begin{array}{cc}I & I \\ * & 0\end{array}\right]$, where $X, U, X_{c}, Y, Y_{c} \in \Re^{n \times n}$ and the dimensions of $\tilde{T}$ and its submatrices are implicitly determined by $\tilde{P}$. Moreover, the following changes of variables are carried out

$$
V=U B_{c}, \quad P=Y^{-1}, \quad S=A_{c}{ }^{T} U^{\prime},
$$

where the dimensions of matrices $V, P$ and $S$ are implicity determined by the transformations.

Now, the set of BMIs (59)-(60) can be transformed into a set of LMIs. For that, multiply $\tilde{P},(59)$ and (60) on the right and the left by $T$, $\operatorname{diag}[T, I]$ and $\operatorname{diag}[T, T]$, respectively; introduce the new variables $V, P$ and $S$, and simplify the expressions by algebraic manipulations, remembering that $\tilde{P} \tilde{P}^{-1}=I$. The resulting set of LMIs are given by

$$
\left[\begin{array}{cc}
P & P \\
* & X
\end{array}\right] \succ 0,\left[\begin{array}{ccc}
D_{11} & D_{12} & D_{13} \\
* & D_{22} & D_{23} \\
* & * & D_{33}
\end{array}\right] \prec 0
$$

$$
\left[\begin{array}{cccc}
N_{11} & N_{12} & N_{13} & N_{14} \\
* & N_{22} & N_{23} & N_{24} \\
* & * & N_{33} & N_{34} \\
* & * & * & N_{44}
\end{array}\right] \prec 0,
$$

where, $D_{11}=\bar{A}_{0 i}^{T} P+P \bar{A}_{0 i}+G_{i}^{T} G_{i}+2 \alpha P, D_{12}=P A_{0 i}+$ $\bar{A}_{0 i}^{T} X+C_{y i}^{T} V^{T}+S+G_{i}^{T} G_{i}+2 \alpha P, D_{13}=P F_{i}, D_{22}=$ $A_{0 i}^{T} X+X A_{0 i}+V C_{i}+C_{i}^{T} V^{T}+G_{i}^{T} G_{i}+2 \alpha X, D_{23}=X F_{i}$, $D_{33}=-I, N_{11}=\left(\bar{A}_{0 i}^{T} P+P \bar{A}_{0 i}\right) \sin (\delta), N_{12}=\left(\bar{A}_{0 i}^{T} X+\right.$ $P \bar{A}_{0 i}+C_{i}^{T} V^{T}+S \sin (\delta), N_{13}=\left(\bar{A}_{0 i}^{T} P-P \bar{A}_{0 i}\right) \cos (\delta)$, $N_{14}=\left(\bar{A}_{0 i}^{T} X-P A_{0 i}+C_{i}^{T} V^{T}+S\right) \cos (\delta), N_{22}=\left(A_{0 i}^{T} X+\right.$ $\left.X A_{0 i}+C_{i}^{T} V^{T}+V C_{i}\right) \sin (\delta), N_{24}=\left(A_{0 i}^{T} X-X A_{0 i}+\right.$ $\left.C_{i}^{T} V^{T}-V C_{i}\right) \cos (\delta), N_{23}=N_{14}^{\prime}, N_{33}=N_{11}, N_{34}=N_{12}$, $N_{44}=N_{22}, \bar{A}_{0 i}=A_{0 i}+B_{i} C_{c}, i=1, \ldots, n_{p}$

Solving this set of LMIs in the matrices variables $V, P, S$ and $X$, the matrices $A_{c}$ and $B_{c}$ of the controller (remembering that matrix $C_{c}$ must be pre-specified) can be calculated by

$$
B_{c}=U^{-1} V, A_{c}=U^{-1} S^{\prime},
$$

where $U=P-X$. The success in solving this LMI problem depends, obviously, in a proper choice of matrix $C_{c}$. In this paper, we calculate this matrix by setting up a state feedback gain $K$, in which the control law $u(t)=K x(t)$ stabilizes the system (5) and fulfills the constraints discussed in section 4. This matrix $K$ can be found by solving the following LMIs in the matrix variables L and Y (Ramos et al., 2004):

$$
Y \succ 0,\left[\begin{array}{cc}
Q_{11} & Q_{12} \\
* & Q_{22}
\end{array}\right] \prec 0,\left[\begin{array}{cc}
R_{11} & R_{12} \\
* & R_{22}
\end{array}\right] \prec 0,
$$

where, $Q_{11}=Y A_{0 i}^{T}+A_{0 i} Y+L^{T} B_{i}^{T}+B_{i} L+2 \alpha Y$, $Q_{12}=Y G_{i}^{T}, Q_{22}=-I, R_{11}=\left(Y A_{0 i}^{T}+A_{0 i} Y+L^{T} B_{i}^{T}+\right.$ $\left.B_{i} L\right) \sin (\delta), R_{12}=\left(Y A_{0 i}^{T}-A_{0 i} Y+L^{T} B_{i}^{T}-B_{i} L\right) \cos (\delta)$, $R_{22}=R_{11}, i=1, \ldots, n_{p}$.

Once we have solved this set of LMIs (65), it is setled $C_{c}:=K$, where $K=L Y^{-1}$.

It is important to emphasize that given the non-convex nature of the undepinning BMI, we cannot guarantee that the LMI problem that is created by setting the matrix $\mathrm{Cc}$ obtained from the solution of the state feedback problem will always have a solution. Recent papers, however have shown that this heuristics for calculation of matrix $C_{c}$ provides satisfactory results for the overall design procedure, as seen, for example, in (Ramos et al., 2004; Ramos et al., 2005; Kuiava et al., 2009).

\section{TESTS AND RESULTS}

Consider the following nonlinear system:

$$
\begin{aligned}
\dot{x}_{1}(t)= & -1.35 x_{2}(t)-0.56 x_{3}(t)+0.08 x_{1}(t) \sin \left(x_{2}(t)\right) \\
& +0.44 \sin ^{2}\left(x_{2}(t)\right)+0.01 \sin \left(x_{2}(t)\right) \sin \left(x_{3}(t)\right)+
\end{aligned}
$$




$$
\begin{aligned}
& 0.22 \sin ^{3}\left(x_{2}(t)\right)+1.35 u(t) \\
\dot{x}_{2}(t)= & 2.8 x_{1}(t)-0.45 x_{2}(t)-0.02 x_{3}(t)- \\
& 0.05 \sin ^{2}\left(x_{2}(t)\right)+0.11 \sin \left(x_{2}(t)\right) \sin \left(x_{3}(t)\right)- \\
& 0.05 \sin ^{3}\left(x_{3}(t)\right)+0.7 u(t) \\
\dot{x}_{3}(t)= & x_{1}(t)+\sin ^{2}\left(x_{2}(t)\right) \cos \left(x_{3}(t)\right) \\
y(t)= & x_{3}(t)
\end{aligned}
$$

We consider three equilibrium points of interest: $x_{e 1}=$ $\left[\begin{array}{lll}0 & 0 & 0\end{array}\right]^{T}, x_{e 2}=\left[\begin{array}{lll}0.3074 & 2.1277 & -4.2718\end{array}\right]^{T}$ and $x_{e 3}=$ $\left[\begin{array}{lll}-0.034 & -0.5368 & 1.4405\end{array}\right]^{T}$. The Jacobian matrices $A_{01}$, $A_{02}$ and $A_{03}$ were calculated via Taylor series expansion of system (66)-(68) around the equilibrium points $x_{e 1}, x_{e 2}$ and $x_{e 3}$, respectively. They are:

$$
\begin{aligned}
& A_{01}=\left[\begin{array}{ccc}
0 & -1.35 & -0.56 \\
2.8 & -0.45 & -0.02 \\
1 & 0 & 0
\end{array}\right], \\
& A_{02}=\left[\begin{array}{ccc}
0.07 & -2.01 & -0.56 \\
2.8 & -0.46 & -0.01 \\
1 & 0.38 & -0.65
\end{array}\right] \\
& A_{03}=\left[\begin{array}{ccc}
-4.09 & -1.58 & -0.56 \\
2.8 & -0.31 & -0.05 \\
1 & -0.11 & -0.26
\end{array}\right] .
\end{aligned}
$$

An eigenvalue analysis shows that these three equilibrium points are all locally asymptotically stable. Our goal is then to design a dynamic output feedback controller that improves the decay rate of the system trajectories, as well as, the damping ratio of these trajectories as they are approaching the equilibrium points. For that, we first modelled the nonlinear system (66)-(68) via three NLDIs in the form (5), each one describing a certain neighborhood of the points $x_{e 1}, x_{e 2}$ and $x_{e 3}{ }^{1}$.

Let us discuss the construction of an NLDI model describing a certain state-space region of the studied nonlinear system containing the equilibrium point at the origin. Then, the same approach was applied to determine the other NLDIs associated to the points $x_{e 2}$ and $x_{e 3}$. Applying the mean value theorem to the nonlinear system (66)-(68) with respect to $x_{e 1}$, we have a reformulation of it in the form of the following LPV system

\footnotetext{
${ }^{1}$ Section 3.5 provided a numerical example where all the steps of the modeling procedure were discussed in details. In the example of this section, our focus is on the control problem. Hence, we omit some details about the application of modeling procedure to the nonlinear system (66)-(68).
}

$$
\begin{aligned}
& {\left[\begin{array}{c}
\dot{x}_{1}(t) \\
\dot{x}_{2}(t) \\
\dot{x}_{3}(t)
\end{array}\right]=} \\
& =\left[\begin{array}{ccc}
\theta_{11}(t) & -1.35+\theta_{12}(t) & -0.56+\theta_{13}(t) \\
2.8 & -0.45+\theta_{22}(t) & -0.02+\theta_{23}(t) \\
1 & \theta_{32}(t) & \theta_{33}(t)
\end{array}\right]\left[\begin{array}{l}
x_{1}(t) \\
x_{2}(t) \\
x_{3}(t)
\end{array}\right] \\
& +\left[\begin{array}{c}
1.35 \\
0.7 \\
0
\end{array}\right] u(t),
\end{aligned}
$$

where $\theta_{i j}: \Re_{+} \rightarrow\left[\underline{h}_{i j}, \bar{h}_{i j}\right], i, j=1, \ldots, 3$.

The bounds $\underline{h}_{i j}$ and $\bar{h}_{i j}$ were specified by analysing the mathematical expressions of the functions $h_{i j}(t)$ (which are calculated by (9)) considering the following state-space region:

$$
\begin{aligned}
X_{1}:= & \left\{\left[\begin{array}{lll}
x_{1} & x_{2} & x_{3}
\end{array}\right]^{\prime} \in \mathbb{R}^{3} \mid-0.25 \leq x_{1} \leq 0.25\right. \\
& \left.-0.25 \leq x_{2} \leq 0.30,-0.3 \leq x_{3} \leq 0.3\right\}
\end{aligned}
$$

The region $X_{1}$ was defined by considering it as being the operating range with respect to the equilibrium point at the origin. Thus, we specified the following bounds for the nonzero functions $h_{i j}(t): \underline{h}_{11}=-0.020, \bar{h}_{11}=0.024, \underline{h}_{12}=$ $-0.236, \bar{h}_{12}=0.316, \underline{h}_{13}=-0.0025, \bar{h}_{13}=0.003, \underline{h}_{22}=-0.09$, $\bar{h}_{22}=0.086, \underline{h}_{23}=-0.05, \bar{h}_{23}=0.055, \underline{h}_{32}=-0.48$, $\bar{h}_{32}=0.56, \underline{h}_{33}=-0.025$ and $\bar{h}_{33}=0.025$.

The LPV system (71) has 7 non-zero functions $h_{i j}(t)$, which means that the corresponding set $\Omega_{P L D I}$ has 128 vertices (as defined by (16)). To complete the procedure, we have to calculate matrices $F_{1}$ and $G_{1}$. Different from the numerical example of section 3.5, all the equations of the system (66)(68) are nonlinear. Hence, none of the elements of $F_{1}$ was forced to be equal to zero. The dimension of this matrix $F_{1}$ was settled to $3 \times 1$. This dimension was chosen instead of $3 \times 2$ or $3 \times 3$ in order to reduce the number of elements to be calculated by the LMI optimization problem. The dimension of matrix $G_{1}$ was chosen to be $3 \times 3$.

By especifying these dimensions to matrices $F_{1}$ and $G_{1}$, matrices $W$ and $V$ were defined as being a $1 \times 3$ full row rank matrix and a $3 \times 3$ symmetric matrix, respectively. From the solution of the optimization problem suggested in section 3.3 using the SeDuMi solver (Sturm, 1999) in conjunction with YALMIP (Lofberg, 2004) it was possible to obtain the following matrices

$F_{1}=\left[\begin{array}{l}72.78 \\ 20.65 \\ 29.03\end{array}\right], G_{1}=\left[\begin{array}{ccc}1.7 \cdot 10^{-3} & 6.8 \cdot 10^{-4} & 6.8 \cdot 10^{-4} \\ 0 & 1.6 \cdot 10^{-3} & 4.4 \cdot 10^{-4} \\ 0 & 0 & 1.5 \cdot 10^{-3}\end{array}\right]$ 
The NLDIs describing the system dynamics around the equilibrium points $x_{e 2}$ and $x_{e 3}$ were calculated considering, respectively, the following regions:

$$
\begin{aligned}
X_{2}:= & \left\{\left[\begin{array}{lll}
x_{1} & x_{2} & x_{3}
\end{array}\right]^{\prime} \in \mathbb{R}^{3} \mid 0.0075 \leq x_{1} \leq 0.5074\right. \\
& \left.1.93 \leq x_{2} \leq 2.53,-6.37 \leq x_{3} \leq-4.12\right\} \\
X_{3}:= & \left\{\left[\begin{array}{ll}
x_{1} & x_{2} \\
x_{3}
\end{array}\right]^{\prime} \in \mathbb{R}^{3} \mid-0.374 \leq x_{1} \leq 0.176\right. \\
& \left.-0.7368 \leq x_{2} \leq-0.1688,-0.71 \leq x_{3} \leq 1.595\right\}
\end{aligned}
$$

The resulting set of NLDIs are given in the form

$$
\begin{aligned}
\dot{x}^{(i)}(t) & =\left(A_{0 i}+F_{i} E_{i}(t) G_{i}\right) x^{(i)}(t)+B u(t), \\
y(t) & =C x^{(i)}(t),
\end{aligned}
$$

where $i=1,2,3$ and $x^{(i)}(t)=x(t)-x_{e i}$. In addition, $A_{01}$, $A_{02}$ and $A_{03}$ are given by (70), respectively; $F_{1}$ and $G_{1}$ by (73) and the other matrices are

$F_{2}=\left[\begin{array}{l}5.44 \\ 7.22 \\ 25.4\end{array}\right], G_{2}=\left[\begin{array}{ccc}4.9 \cdot 10^{-3} & 4.4 \cdot 10^{-4} & 3.8 \cdot 10^{-3} \\ 0 & 5.2 \cdot 10^{-3} & 3.2 \cdot 10^{-4} \\ 0 & 0 & 5.1 \cdot 10^{-3}\end{array}\right]$,
$F_{3}=\left[\begin{array}{l}2.58 \\ 24.2 \\ 36.2\end{array}\right], G_{3}=\left[\begin{array}{ccc}5.1 \cdot 10^{-3} & 4.7 \cdot 10^{-4} & 4.6 \cdot 10^{-3} \\ 0 & 5.4 \cdot 10^{-3} & 4.1 \cdot 10^{-4} \\ 0 & 0 & 5.3 \cdot 10^{-3}\end{array}\right]$.

Finally, matrices $B$ and $C$ are directly determined by looking the nonlinear equations (66)-(69).

Now, let us estimate the region of attraction $D_{1}$ of the equilibrium point $x_{e 1}$ of the nonlinear system (66)-(69) using the corresponding NLDI model previously calculated. This was done by using Lemma 5. As already shown in section 3.5 for the numerical example 1, we have first to describe the set $X_{1}$ in the form of (2). This description can be obtained using the same procedure adopted for that example of section 3.5. As a result, we have a set $X_{1}$ described in the form given by (2), where

$$
\begin{aligned}
& a_{1}=\left[\begin{array}{lll}
2.00 & -2.00 & 0
\end{array}\right], a_{2}=\left[\begin{array}{lll}
0 & -1.64 & 1.97
\end{array}\right], \\
& a_{3}=\left[\begin{array}{lll}
-2.00 & -2.00 & 0
\end{array}\right], a_{4}=\left[\begin{array}{lll}
0 & -1.64 & -1.97
\end{array}\right. \\
& a_{5}=\left[\begin{array}{lll}
-1.64 & 0 & -1.97
\end{array}\right], a_{6}=\left[\begin{array}{lll}
-1.64 & 1.97 & 0
\end{array}\right] \text {, } \\
& a_{7}=\left[\begin{array}{lll}
0 & 1.67 & 1.67
\end{array}\right], a_{8}=\left[\begin{array}{lll}
1.64 & 1.97 & 0
\end{array}\right] \text {, } \\
& a_{9}=\left[\begin{array}{lll}
0 & 1.67 & -1.67
\end{array}\right], a_{10}=\left[\begin{array}{lll}
1.64 & 0 & -1.97
\end{array}\right] \text {, } \\
& a_{11}=\left[\begin{array}{lll}
1.64 & 0 & 1.97
\end{array}\right], a_{12}=\left[\begin{array}{lll}
-1.64 & 0 & 1.97
\end{array}\right] \text {. }
\end{aligned}
$$

Figure 4 shows the ellipsoid $D_{1}$ and the state-space region $X_{1}$ defined as (72).

Now, our goal is to design a dynamic output feedback controller to the nonlinear system (66)-(69) using a description of it in the form of (76)-(77). A decay rate of 0.01 and a minimum damping ratio equal to $15 \%$ were imposed as design objectives to the controlled system. The SeDuMi solver,

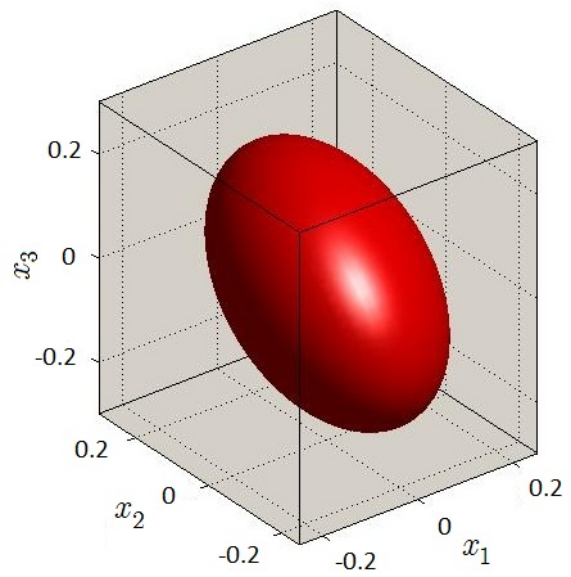

Figure 4: Region $X_{1}$ (the box bounded by the faces in gray) and the estimation of the region of attraction $D_{1}$ (in red) for the equilibrium point at the origin.

used in conjunction with YALMIP, was used to solve the set of LMIs related to this control problem. The calculated controller is given by

$$
\begin{array}{r}
A_{c}=\left[\begin{array}{ccc}
-26.38 & -12.85 & -260.21 \\
-105.5 & -89.36 & -90.68 \\
-4.17 & -3.18 & -110.22
\end{array}\right], \\
B_{c}=\left[\begin{array}{c}
260.5 \\
98.76 \\
110.5
\end{array}\right], \\
C_{c}=\left[\begin{array}{lll}
-9.55 & -5.25 & 0.58
\end{array}\right] .
\end{array}
$$

The performance of the controlled system was verified via nonlinear simulations. Figs. 5, 6 and 7 show the response of variables $x_{1}(t), x_{2}(t)$ and $x_{3}(t)$, respectively, with respect to different initial conditions. It is interesting to observe that

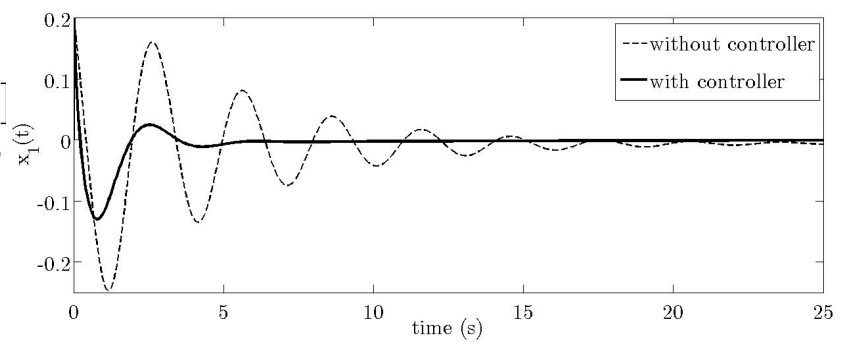

Figure 5: Response of $x_{1}(t)$ for the initial condition $x_{0}=$ $\left[\begin{array}{lll}0.2 & 0.3 & 0.1\end{array}\right]^{T}$ applied at $t=0$.

the trajectory of the controlled system is less oscillatory and approaches the equilibrium faster than the trajectory of the 


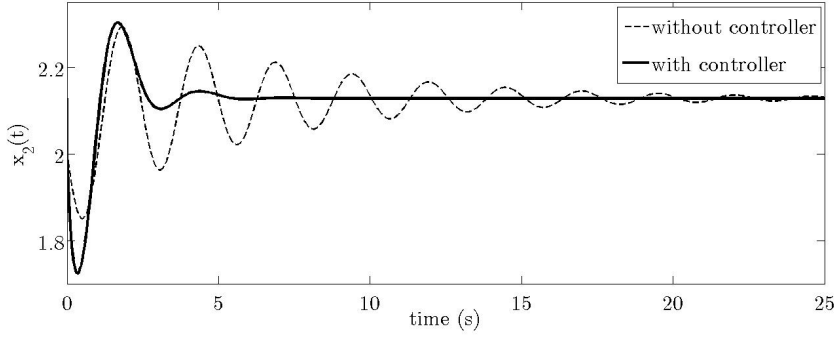

Figure 6: Response of $x_{2}(t)$ for the initial condition $x_{0}=$ $\left[\begin{array}{lll}0.1 & 2.0 & -4.0\end{array}\right]^{T}$ applied at $t=0$.

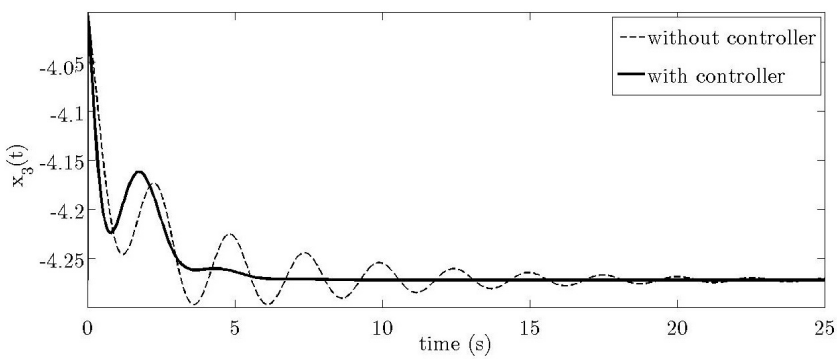

Figure 7: Response of $x_{3}(t)$ for the initial condition $x_{0}=$ $\left[\begin{array}{lll}0.2 & 2.2 & -4\end{array}\right]^{T}$ applied at $t=0$.

open-loop system. This result shows the effectiveness of the designed controller.

The region of attraction of the equilibrium point $\tilde{x}_{e_{1}}$ of the closed-loop nonlinear system, where $\tilde{x}_{e_{1}}=\left[\begin{array}{llll}x_{e_{1}}^{\prime} & 0 & 0 & 0\end{array}\right]^{\prime}$, was also estimated using the corresponding NLDI model in the form of (58). For that, we defined the ellipsoid $\tilde{D}_{1}:=$ $\left\{\tilde{x} \in \mathbb{R}^{6} \mid \tilde{x}^{\prime} \tilde{P}_{1} \tilde{x} \leq 1\right\}$.

Figure 8 shows the ellipsoid $\tilde{D}_{1}$, considering the state of the controller equal to zero. This plot corresponds to the cut of the actual estimate of ellipsoid $\tilde{D}_{1}$ in the hyperplane defined by the system states. Hence, from the result shown in Figure 8 , the effectiveness of the designed controller for the nonlinear system (66)-(69) is guaranteeded within the region $\tilde{D}_{1}$.

\section{CONCLUSION}

In the first part of this paper, a method to calculate the parameters of an NLDI model was presented. The objective is to obtain a suitable linear representation of a nonlinear system for control purposes, in such a way that a linear controller can be designed to guarantee some desired features for the nonlinear closed-loop system. In the second part, a robust control design method, written in terms of LMIs was presented, in order to design a linear dynamic output feedback

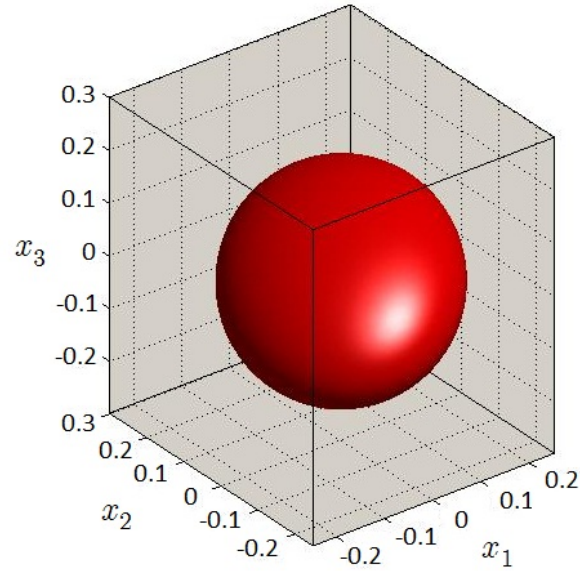

Figure 8: Region $X_{1}$ (the box bounded by the faces in gray) and the estimation of the region of attraction $\tilde{D}_{1}$ for the closed-loop nonlinear system (in red) with respect to the equilibrium point at the origin.

controller for a nonlinear system using the NLDI model obtained by the approah proposed in the first part.

The numerical examples presented in the previous section have shown the effectiveness of the modeling and control approach proposed in this paper. However, it is important to emphasize that the application of the modeling procedure may be restricted to small and medium size systems, once that the number of vertices of the obtained PLDI may become excessively high as the number of system nonlinearities increases. With respect to the proposed control design, one of its difficulties is the requirement, due to the nature of the control problem formulation, that the order of the designed LDOF controller must be equal to the order of the system to be controlled. In order to achieve the goal of producing low order controllers (in the cases where the dimension of the system is sufficiently high), the control design may be combined with a final step of model order reduction.

The design objectives targeted in this paper were a minimum decay rate for all possible system trajectories within the operating region of interest and a minimum damping ratio to the oscillation modes of the state matrix of the closed-loop system. These design objectives are very important for the problem of robust damping control in power systems, for example, and an application of the approach proposed in this paper to such a problem is the next predicted step of this research. It is important to point out, however, that other practical requirements can be included in the control problem formulation, such as the minimization of an output energy function or restrictions on the controller bandwidth. These are also 
possible extensions of this research foreseen to the sequence of this research.

\section{ACKNOWLEDGE}

The authors would like to thank the reviewers for their helpful comments and suggestions.

The authors would like also to thank Prof. Luis Fernando Costa Alberto for providing the Matlab routines used for the estimates of the regions of attractions in examples 1 and 2 .

\section{REFERENCES}

Basler, M. J. and Schaefer, R. C. (2008). Understanding power system stability, IEEE Trans. Indus. Appl. 44(2): 463-474.

Bernard, F., Dufour, F. and Bertrand, P. (1997). On the jlq problem with uncertainty, IEEE Trans. Automat. Contr. 42(6): 869-872.

Boyd, S., Ghaoui, L. E., Feron, E. and Balakrishnam, V. (1994). Linear Matrix Inequalities in System and Control Theory, Society for industrial and applied mathematics.

Chen, B. S., Lee, C. H. and Chang, Y. C. (1996). H $_{\infty}$ tracking design of linear systems: adaptative fuzzy approach, IEEE Trans. Fuzzy Syst. 4(5): 32-43.

Chiali, M., Gahinet, P. and Apkarian, P. (1999). Robust pole placement in lmi regions, IEEE Trans. Automat. Contr. 44(12): 2257-2270.

Coutinho, D. F. and da Silva Jr., J. M. G. (2010). Computing estimates of the region of attraction for rational control systems with saturating actuators, IET Control Theory Applications 4(3): 315-325.

de Oliveira, M. C., Geromel, J. C. and Bernussou, J. (2000). Design of dynamic output feedback decentralized controllers via a separation procedure, Int. J. Contr. 73(5): 371-381.

de Oliveira, R. V., Kuiava, R., Ramos, R. A. and Bretas, N. G. (2009). Automatic tuning method for the design of supplementary damping controllers for flexible alternating current transmission system devices, IET Gen., Trans. \& Distrib. 3(10): 919-929.

Folcher, J. P. and Ghaoui, E. L. (1994). State-feedback design via linear matrix inequalities: application to a benchmark problem, Proc. of the IEEE Conf. on Contr. Appl.
Hossain, J., Pota, H. R., Ugrinovskii, V. and Ramos, R. A. (2009). A novel STATCOM control to augment LVRT of fixed speed wind generators, Proc. of the IEEE Conf. on Dec. and Contr.

$\mathrm{Hu}, \mathrm{T}$. (2007). Nonlinear control design for linear differential inclusions via convex hull of quadratics, Automatica 43(4): 685-692.

Hu, X. B. and Chen, W. H. (2007). Model predictive control of nonlinear systems: stability region and feasible initial control, Int. J. Automation and Comput. 4(2): 195202.

Kiyama, T. and Iwasaki, T. (2000). On the use of multi-loop circle criterion for saturating control synthesis, System and Control Letters 41: 105-114.

Kuiava, R., Ramos, R. A. and Bretas, N. G. (2009). Robust control methodology for the design of supplementary damping controllers for FACTS devices, Revista Controle \& Automao 20(2): 192-205.

Lofberg, J. (2004). Yalmip: a toolbox for modeling and optimization in matlab, Proc. of the CACSD Conf., Taipei, Taiwan.

*http://control.ee.ethz.ch/ joloef/yalmip.php

Montagner, V., Oliveira, R. C. L. F. and Peres, P. L. D. (2010). Relaxaes convexas de convergncia garantida para o projeto de controladores para sistemas nebulosos de takagi-sugeno, Revista Controle \& Automao 22(1): 82-95.

Mozelli, L. A., Palhares, R. M., de Avellar, G. S. C. and dos Santos, R. F. (2010). Condies lmis alternativas para sistemas takagi-sugeno via funo de lyapunov fuzzy, $R e$ vista Controle \& Automao 21(1): 96-107.

Orsi, R., Helmke, U. and Moore, J. B. (2006). A newtonlike method for solving rank constrained linear matrix inequalities, Automatica 42(11): 1875-1882.

Polanski, A. (1997). Lyapunov function construction by linear programming, IEEE Trans. Automat. Contr. 42(7): 1013-1016.

Pyatnitskiy, E. S. and Rapoport, L. B. (1996). Criteria of asymptotic stability of differential inclusions and periodic motions of time-varying nonlinear control systems, IEEE Trans. Circ. Syst. I 43(3): 219-229.

Ramos, R. A., Alberto, L. F. C. and Bretas, N. G. (2004). A new methodology for the coordinated design of robust decentralized power system damping controllers, IEEE Trans. Power Syst. 19(1): 444-454. 
Ramos, R. A., Martins, A. C. P. and Bretas, N. G. (2005) Improved methodology for the design of power system damping controllers, IEEE Trans. Power Syst. 20(4): 1938-1945.

Rohr, E. R., Pereia, L. F. A. and Coutinho, D. F. (2009). Robustness analysis of nonlinear systems subject to state feedback linearization, SBA Controle e Automao 20(4): 482-489.

Silva, S. and Junior, V. L. (2006). Active flutter suppression in a 2-d airfoil using linear matrix inequalities techniques, J. Braz. Soc. Mech. Sci. Eng. 28(1): 84-93.

Sturm, J. F. (1999). Using SeDuMi 1.02, a Matlab toolbox for optimization over symmetric cones, Optimization Methods and Software 11(1): 625-653.

Tognetti, E. S. and Oliveira, V. A. (2010). Fuzzy pole placement based on piecewise lyapunov functions, Int. J. Robust and Nonlin. Contr. 20(1): 571-578.
Topcu, U. and Packard, A. (2009). Local stability analysis for uncertain nonlinear systems, IEEE Trans. Automat. Contr. 54(5): 1042-1047.

Vidyasagar, M. (1993). Nonlinear systems analysis, Englewood Cliffs, N.J: Prentice Hall.

Xie, L. and de Souza, C. E. (1992). Robust control for linear systems with norm-bounded time-varying uncertainty, IEEE Trans. Automat. Contr. 37(8): 1188-1191.

Yfoulis, C. A. and Shorten, R. (2004). A numerical technique for the stability analysis of linear switched systems, Int. J. Contr. 77(11): 1019-1039.

Zemouche, A., Boutayeb, M. and Bara, G. (2005). Observer design for nonlinear systems: an approach based on the differential mean value theorem, Proc. of the IEEE Conf. on Dec. and Contr. 